\title{
Recent work connected with the Kakeya problem
}

\author{
Thomas Wolff \\ Department of Mathematics \\ 253-37 Caltech \\ Pasadena, Ca 91125 USA \\ wolff@cco.caltech.edu
}

A Kakeya set in $\mathbb{R}^{n}$ is a compact set $E \subset \mathbb{R}^{n}$ containing a unit line segment in every direction, i.e.

$$
\forall e \in S^{n-1} \exists x \in \mathbb{R}^{n}: x+t e \in E \forall t \in\left[-\frac{1}{2}, \frac{1}{2}\right]
$$

where $S^{n-1}$ is the unit sphere in $\mathbb{R}^{n}$. This paper will be mainly concerned with the following issue, which is still poorly understood: what metric restrictions does the property (1) put on the set $E$ ?

The original Kakeya problem was essentially whether a Kakeya set as defined above must have positive measure, and as is well-known, a counterexample was given by Besicovitch in 1920. A current form of the problem is as follows:

\section{Open question 1: Must a Kakeya set in $\mathbb{R}^{n}$ have Hausdorff dimension $n$ ?}

When $n=2$, the answer is yes; this was proved by Davies [19] in 1971. Recent work on the higher dimensional question began with [7]. If $\operatorname{dim} E$ denotes the Hausdorff dimension then the bound $\operatorname{dim} E \geq \frac{n+1}{2}$ can be proved in several ways and may have been known prior to [7], although the author has not been able to find a reference. The recent work [7], [60] has led to the small improvement $\operatorname{dim} E \geq \frac{n+2}{2}$. We will discuss this in section 2 below.

Question 1 appears quite elementary, but is known to be connected to a number of basic open questions in harmonic analysis regarding estimation of oscillatory integrals. This is a consequence of C. Fefferman's solution of the disc multiplier problem [23] and work of Cordoba (e.g. [18]) and Bourgain (e.g. [7], [9], [10]). We will say something about these interrelationships in section 4 . There is also a long history of applications of Kakeya sets to construct counterexamples in pointwise convergence questions; we will not discuss this here, but see e.g. [25] and [54].

For various reasons it is better to look also at a more quantitative formulation in terms of a maximal operator. If $\delta>0, e \in S^{n-1}, a \in \mathbb{R}^{n}$ then we define

$$
T_{e}^{\delta}(a)=\left\{x \in \mathbb{R}^{n}:|(x-a) \cdot e| \leq \frac{1}{2},\left|(x-a)^{\perp}\right| \leq \delta\right\}
$$


where $x^{\perp}=x-(x \cdot e)$ e. Thus $T_{e}^{\delta}(a)$ is essentially the $\delta$-neighborhood of the unit line segment in the $e$ direction centered at $a$. If $f: \mathbb{R}^{n} \rightarrow \mathbb{R}$ then we define its Kakeya maximal function $f_{\delta}^{*}: S^{n-1} \rightarrow \mathbb{R}$ via

$$
f_{\delta}^{*}(e)=\sup _{a \in \mathbb{R}^{n}} \frac{1}{\left|T_{e}^{\delta}(a)\right|} \int_{T_{e}^{\delta}(a)}|f|
$$

This definition is due to Bourgain [7]. It is one of several similar maximal functions that have been considered, going back at least to [18].

Open question 2: Is there an estimate

$$
\forall \epsilon>0 \exists C_{\epsilon}:\left\|f_{\delta}^{*}\right\|_{L^{n}\left(S^{n-1}\right)} \leq C_{\epsilon} \delta^{-\epsilon}\|f\|_{n} \forall f
$$

Roughly speaking, this question is related to question 1 in the same way as the HardyLittlewood maximal theorem is related to Lebesgue's theorem on points of density. As was observed by Bourgain [7], an affirmative answer to question 2 implies an affirmative answer to question 1; see Lemma 1.6 below. Once again, when $n=2$ it is well known that the answer to question 2 is affirmative, [18] and [7]. In higher dimensions, partial results are known parallelling the results on question 1.

Questions 1 and 2 clearly have a combinatorial side to them, and the point of view we will adopt here is to try to approach the combinatorial issues directly using ideas from the combinatorics literature. In this connection let us mention a basic principle in graph theory (the "Zarankiewicz problem"; see [5], [24], [40] for this and generalizations): fix $s$ and suppose that $\left\{a_{i j}\right\}_{i=1}^{n} \underset{j=1}{m}$ is an $n \times m(0,1)$ matrix with no $s \times s$ submatrix of 1's. Then there is a bound

$$
\left|\left\{(i, j): a_{i j}=1\right\}\right| \leq C_{s} \min \left(m n^{1-\frac{1}{s}}+n, n m^{1-\frac{1}{s}}+m\right)
$$

To see the relationship between this sort of bound and Kakeya, just note that if $\left\{\ell_{j}\right\}_{j=1}^{m}$ are lines and $\left\{p_{i}\right\}_{i=1}^{n}$ are points, then the "incidence matrix"

$$
a_{i j}= \begin{cases}1 & \text { if } p_{i} \in \ell_{j} \\ 0 & \text { if } p_{i} \notin \ell_{j}\end{cases}
$$

will contain no $2 \times 2$ submatrix of 1's, since two lines intersect in at most one point. Much of what we will say below will have to do with attempts to modify this argument, and also more sophisticated arguments in incidence geometry (e.g. [17]) to make them applicable to "continuum" problems such as Kakeya.

There are several difficulties with such an approach. It is sometimes unclear whether applying the combinatorial techniques in the continuum should be simply a matter of extra technicalities or whether new phenomena should be expected to occur, and furthermore 
many of the related discrete problems are regarded as being very difficult. A classical example is the Erdos unit distance problem (see [17] and [40]) and other examples will be mentioned in section 3 .

Of course, much work has been done in the opposite direction, applying harmonic analysis techniques to questions of a purely geometrical appearance. A basic example is the spherical maximal theorem of Stein [51], and various Strichartz type inequalities as well as the results on the distance set problem in [22], [11] are also fairly close to the subject matter of this paper. However, we will not present any work of this nature here.

The paper is organized as follows. In section 1 we discuss the two dimensional Kakeya problem, in section 2 we discuss the higher dimensional Kakeya problem and in section 3 we discuss analogous problems for circles in the plane. Finally in section 4 we discuss the Fefferman construction and a related construction of Bourgain [9] which connects the Kakeya problem also to estimates of Dirichlet series. Section 4 contains several references to the recent literature on open problems regarding oscillatory integrals, but it is not a survey. Further references are in [10], [58], and especially [52].

We have attempted to make the presentation self-contained insofar as is possible. In particular we will present some results and arguments which are known or almost known but for which there is no easy reference.

The author is grateful for the opportunity to speak at the conference and to publish this article.

\section{List of notation}

$[\alpha]$ : greatest integer less than or equal to $\alpha$.

$p^{\prime}$ : conjugate exponent to $p$, i.e. $p^{\prime}=\frac{p}{p-1}$.

$D(x, r)$ : the disc with center $x$ and radius $r$.

$|E|$ : Lebesgue measure or cardinality of the set $E$, depending on the context.

$E^{c}$ : complement of $E$.

$\operatorname{dim} E$ : Hausdorff dimension of $E$.

$H_{s}(E): s$-dimensional Hausdorff content of $E$, i.e. $H_{s}(E)=\inf \left(\sum_{j} r_{j}^{s}: E \subset \cup_{j} D\left(x_{j}, r_{j}\right)\right)$ $T_{e}^{\delta}(a): \delta$-tube in the $e$ direction centered at $a$, as defined in the introduction. Sometimes we will also use the notation $T_{e}^{\delta}$; this means any tube of the form $T_{e}^{\delta}(a)$ for some $a \in \mathbb{R}^{n}$. $C(x, r)$ : circle in $\mathbb{R}^{2}$ (or sphere in $\mathbb{R}^{n}$ ) with center $x$ and radius $r$.

$C_{\delta}(x, r)$ : annular region $\left\{y \in \mathbb{R}^{n}: r-\delta<|y-x|<r+\delta\right\}$.

$x \lesssim y: x \leq C y$ for a suitable constant $C$.

\section{The two dimensional case}

We will start by proving the existence of measure zero Kakeya sets using a variant on the original construction which is quick and is easy to write out in closed form; to the author's knowledge the earliest reference for this approach is Sawyer [42]. A discussion of various other possible approaches to the construction may be found in [21]. 
For expository reasons, we make the following definitions.

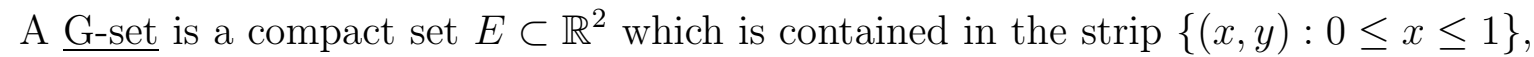
such that for any $m \in[0,1]$ there is a line segment contained in $E$ connecting $x=0$ to $x=1$ with slope $m$, i.e.

$$
\forall m \in[0,1] \exists b \in \mathbb{R}: m x+b \in E \forall x \in[0,1]
$$

If $\ell=\{(x, y): y=m x+b\}$ is a nonvertical line, $\delta>0$, then $S_{\ell}^{\delta} \stackrel{\text { def }}{=}\{(x, y): 0 \leq x \leq 1$ and $|y-(m x+b)| \leq \delta\}$.

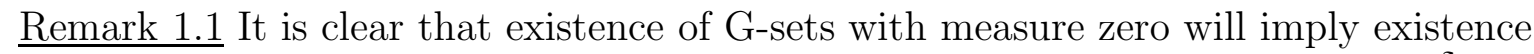
of Kakeya sets with measure zero. Note also that if $\ell$ is a line with slope $m$ then $S_{\ell}^{\delta}$ will contain segments connecting $x=0$ to $x=1$ with any given slope between $m-2 \delta$ and $m+2 \delta$.

We now describe the basic construction, which leads to the slightly weaker conclusion (Lemma 1.2) that there are G-sets with measure $<\epsilon$ for any $\epsilon>0$. It can be understood in terms of the usual sliding triangle picture: start from a right triangle with vertices $(0,0),(0,-1)$ and $(1,0)$; this is clearly a $G$-set. Subdivide it in $N$ "1st stage" triangles by subdividing the vertical side in $N$ equal intervals. Leave the top triangle alone and slide the others upward so that their intersections with the line $x=0$ all coincide. Next, for each of the 1st stage triangles, subdivide it in $N$ 2nd stage triangles, leave the top triangle in each group alone and slide the $N-1$ others upward until the intersections of the $N$ triangles in the group with the line $x=\frac{1}{N}$ all coincide. Now repeat at abscissas $\frac{2}{N}, \frac{3}{N}, \ldots, \frac{N-1}{N}$.

Now we make this precise. Fix a large integer $N$ and let $\mathcal{A}_{N}$ be all numbers in $[0,1)$ whose base $N$ expansion terminates after $N$ digits, i.e.

$$
a \in \mathcal{A}_{N} \Leftrightarrow a=\sum_{j=1}^{N} \frac{a_{j}}{N^{j}} \text { with } a_{j} \in\{0,1, \ldots N-1\}
$$

To each $a \in \mathcal{A}_{N}$ we associate the line segment $\ell_{a}$ connecting the $y$ axis to the line $x=1$ with slope $a$ and $y$ intercept $-\sum_{j=1}^{N} \frac{(j-1) a_{j}}{N^{j+1}}$. Thus

$$
\ell_{a}=\left\{\left(t, \phi_{a}(t)\right): 0 \leq t \leq 1\right\}, \text { where } \phi_{a}(t)=\sum_{j=1}^{N} \frac{(N t-j+1) a_{j}}{N^{j+1}}
$$

Lemma 1.1 For each $t \in[0,1]$ there are an integer $k \in\{1, \ldots, N\}$ and a set of $N^{k-1}$ intervals each of length $2 N^{-k}$, whose union contains the set $\left\{\phi_{a}(t): a \in \mathcal{A}_{N}\right\}$. 
$\underline{\text { Proof }}$ Choose $k$ so that $\frac{k-1}{N} \leq t \leq \frac{k}{N}$. Define $a, b \in \mathcal{A}_{N}$ to be equivalent if $a_{j}=b_{j}$ when $j \leq k-1$. There are $N^{k-1}$ equivalence classes, and if $a$ and $b$ are equivalent then

$$
\begin{aligned}
\left|\phi_{a}(t)-\phi_{b}(t)\right| & =\left|\sum_{j \geq k} \frac{(N t-j+1)\left(a_{j}-b_{j}\right)}{N^{j+1}}\right| \\
& \leq \sum_{j \geq k} \frac{\max (j-k, 1)\left|a_{j}-b_{j}\right|}{N^{j+1}} \\
& \leq \frac{N-1}{N^{k+1}} \sum_{j \geq k} \frac{\max (j-k, 1)}{N^{j-k}} \\
& \leq 2 \frac{N-1}{N^{k+1}}<\frac{2}{N^{k}}
\end{aligned}
$$

when $N$ is large.

Lemma 1.2 Let $N$ be sufficiently large. Then there is a G-set $E \subset[0,1] \times[-1,1]$ which intersects every vertical line in measure $\leq \frac{4}{N}$, in particular $|E| \leq \frac{4}{N}$.

$\underline{\text { Proof We let }}$

$$
E_{N}=\cup_{a \in \mathcal{A}_{N}} S_{\ell_{a}}^{N^{-N}}
$$

Then $E_{N}$ contains segments with all slopes between 0 and 1 , by Remark 1.1. If $t \in[0,1]$, then by Lemma 1.1 there is $k \in\{1, \ldots, N\}$ such that the intersection of $E$ with the line $x=t$ is contained in the union of $N^{k-1}$ intervals of length $2 N^{-k}+2 N^{-N} \leq 4 N^{-k}$. The lemma follows.

Existence of measure zero Kakeya sets now follows by a standard limiting argument, most easily carried out via the following lemma.

Lemma 1.3: For every G-set $E$ and every $\epsilon>0, \eta>0$, there is another $G$-set $F$, which is contained in the $\epsilon$-neighborhood of $E$ and has measure $<\eta$.

Proof Let $\delta$ be small, let $\left\{m_{j}\right\}=\{j \delta\}_{j=0}^{\left[\frac{1}{\delta}\right]}$ and for each $j$, fix a line segment $\ell_{j}=$ $\left\{(x, y): 0 \leq x \leq 1, y=m_{j} x+b_{j}\right\} \subset E$ with slope $m_{j}$ connecting $x=0$ to $x=1$ and form the parallelogram $S_{\ell_{j}}^{\delta}$. Let $A_{j}$ be the affine map from $[0,1] \times[-1,1]$ on $S_{\ell_{j}}^{\delta}, A_{j}(x, y)=$ $\left(x, m_{j} x+b_{j}+\delta y\right)$ and consider $F=\cup_{m} A_{m}\left(E_{N}\right)$ for a large enough $N$; here $E_{N}$ is as in Lemma 1.2. $A_{j}$ maps segments with slope $\mu$ to segments with slope $m+\delta \mu$ so $F$ is a G-set. Clearly it is contained in the $\delta$-neighborhood of $E$. Furthermore $A_{j}$ contracts areas by a factor $\delta$ so $\left|A_{j}\left(E_{N}\right)\right| \leq 4 \frac{\delta}{N}$ for each $j$, hence $|F| \lesssim \frac{1}{N}$. 
Corollary 1.4 There are Kakeya sets with measure zero.

Proof We construct a sequence $\left\{F_{n}\right\}_{n=0}^{\infty}$ of G-sets, and a sequence of numbers $\left\{\epsilon_{n}\right\}_{n=0}^{\infty}$ converging to zero such that the following properties hold when $n \geq 1$; here $F(\epsilon) \stackrel{\text { def }}{=}\{x$ : $\operatorname{dist}(x, F)<\epsilon\}$ is the $\epsilon$-neighborhood of $F$ and $\bar{E}$ is the closure of $E$.

(i) $F_{n}\left(\epsilon_{n}\right) \subset F_{n-1}\left(\epsilon_{n-1}\right)$

(ii) $\left|\overline{F_{n}\left(\epsilon_{n}\right)}\right|<2^{-n}$.

Namely, we take $F_{0}$ to be any G-set, and we set $\epsilon_{0}=1$. If $n \geq 1$ and if $F_{n-1}$ and $\epsilon_{n-1}$ have been constructed then we obtain $F_{n}$ by applying Lemma 1.3 with $\epsilon=\epsilon_{n-1}$ and $\eta=2^{-n}$. Since $F_{n}$ is compact, (i) and (ii) will then hold provided $\epsilon_{n}$ is sufficiently small.

The set $\cap_{n} \overline{F_{n}\left(\epsilon_{n}\right)}$ is then a G-set with measure zero.

Remarks 1.2. The construction above easily gives the following variant (used e.g. in [23]): with $\delta=\frac{1}{10} N^{-N}$, there is a family of disjoint $\delta$-tubes $\left\{T_{e_{j}}^{\delta}\left(x_{j}\right)\right\}_{j=1}^{M} \subset \mathbb{R}^{2}$ where $M \approx \delta^{-1}$ with the property that the union of the translated tubes $T_{e_{j}}^{\delta}\left(x_{j}+2 e_{j}\right)$ has measure $\lesssim \frac{1}{N}$.

Namely, a calculation shows that if $a, b \in \mathcal{A}$ and $a<b$ then $\phi_{a}(1)<\phi_{b}(1)$, i.e. the ordering of the intersection points between the $\ell_{a}$ and the line $x=1$ is the same as the ordering of slopes. Hence if we regard $\ell_{a}$ as extended to a complete line, then no two $\ell_{a}$ 's intersect in the region $x>1$, and in fact in the region $x>2$ any two of them are at least $N^{-N}$ apart. Now for each $a \in \mathcal{A}_{N}$ we form the rectangle $R_{a}$ with length 1 , width $\frac{1}{5} N^{-N}$, axis along the line $\ell_{a}$ and bottom right corner on the line $x=1$. Clearly $R_{a} \subset S_{a}$, so $\cup_{a} R_{a}$ is small by Lemma 1.2. On the other hand, if $R_{a}$ is translated to the right along its axis by distance 2 then the resulting rectangles are disjoint. We may therefore take $\left\{T_{e_{j}}^{\delta}\left(x_{j}\right)\right\}$ to be the set of translated rectangles.

1.3. Analogous statements in higher dimensions may be obtained using dummy variables.

Measure zero Kakeya sets in $\mathbb{R}^{n}$ may be constructed by taking the product of a Kakeya set in $\mathbb{R}^{2}$ with a closed disc of radius $\frac{1}{2}$ in $\mathbb{R}^{n-2}$ (or for that matter with any Kakeya set in $\left.\mathbb{R}^{n-2}\right)$, and a family of roughly $\delta^{-(n-1)}$ disjoint $T_{e}^{\delta}(a)$ 's such that the union of the tubes $T_{e}^{\delta}(a+2 e)$ has small measure may be obtained by taking the products of the tubes in Remark 1.2 with a family of $\delta^{-(n-2)}$ disjoint $\delta$-discs in $\mathbb{R}^{n-2}$.

We now discuss the positive results on questions 1 and 2 in dimension two. Proposition 1.5 was first stated and proved in [7] although a similar result for a related maximal function was proved earlier in [18].

We will work with restricted weak type estimates instead of with $L^{p}$ estimates; this is 
known to be equivalent except for the form of the $\delta^{-\epsilon}$ terms. ${ }^{1}$ We will say (see e.g. [53]) that an operator $T$ has restricted weak type norm $\leq A$, written

$$
\|T f\|_{q, \infty} \leq A\|f\|_{p, 1}
$$

if $\left|\left\{x:\left|T \chi_{E}(x)\right| \geq \lambda\right\}\right| \leq\left(\frac{A|E|^{\frac{1}{p}}}{\lambda}\right)^{q}$ for all sets $E$ with finite measure and all $\lambda \in(0,1]$; here $\chi_{E}$ is the characteristic function of $E$.

Proposition 1.5 The restricted weak type $(2,2)$ norm of the Kakeya maximal operator $f \rightarrow f_{\delta}^{*}$ in $\mathbb{R}^{2}$ is $\lesssim\left(\log \frac{1}{\delta}\right)^{\frac{1}{2}}$.

More explicitly, suppose that $E \subset \mathbb{R}^{2}$ and $\lambda \in(0,1]$. Let $f=\chi_{E}$, and let $\Omega=\{e \in$ $\left.S^{1}: f_{\delta}^{*}(e) \geq \lambda\right\}$. Then

$$
|\Omega| \lesssim \log \frac{1}{\delta} \frac{|E|}{\lambda^{2}}
$$

Proof Let $\theta(e, f)$ be the unoriented angle subtended by the directions $e$ and $f$, i.e. $\theta(e, f)=\arccos (e \cdot f)$. We start by mentioning two trivial but important facts. First, in $\mathbb{R}^{n}$, the intersection of the tubes $T_{e}^{\delta}(a)$ and $T_{f}^{\delta}(b)$ satisfies

$$
\operatorname{diam}\left(T_{e}^{\delta}(a) \cap T_{f}^{\delta}(b)\right) \lesssim \frac{\delta}{\theta(e, f)+\delta}
$$

for any $a$ and $b$ and therefore also

$$
\left|T_{e}^{\delta}(a) \cap T_{f}^{\delta}(b)\right| \lesssim \frac{\delta^{n}}{\theta(e, f)+\delta}
$$

Next, if $\Omega$ is a set on the unit sphere $S^{n-1} \subset \mathbb{R}^{n}$ and if $\delta>0$ then the $\delta$-entropy $\mathcal{N}_{\delta}(\Omega)$ (maximum possible cardinality $M$ for a $\delta$-separated subset $\left\{e_{j}\right\}_{j=1}^{M} \subset \Omega$ ) satisfies

$$
\mathcal{N}_{\delta}(\Omega) \gtrsim \frac{|\Omega|}{\delta^{n-1}}
$$

Now we assume $n=2$ and give the proof of the proposition. Fix a $\delta$-separated $\left\{e_{j}\right\}_{j=1}^{M} \subset$ $\Omega$, where $M \gtrsim \frac{|\Omega|}{\delta}$. For each $j$, there is a tube $T_{j}=T_{e_{j}}^{\delta}\left(a_{j}\right)$ with $\left|T_{j} \cap E\right| \geq \lambda\left|T_{j}\right| \approx \lambda \delta$. Thus

$$
M \lambda \delta \lesssim \sum_{j}\left|T_{j} \cap E\right|
$$

\footnotetext{
${ }^{1}$ We work with restricted weak type estimates for expository reasons only. We believe this makes the results more transparent; however, it is well known that actually $\left\|f_{\delta}^{*}\right\|_{L^{2}\left(S^{1}\right)} \lesssim\left(\log \frac{1}{\delta}\right)^{\frac{1}{2}}\|f\|_{2}$. The latter estimate is proved in [7] and also follows from the proof below, plus duality, as in [18].
} 


$$
\begin{aligned}
& =\int_{E} \sum_{j} \chi_{T_{j}} \\
& \leq|E|^{\frac{1}{2}}\left\|\sum_{j} \chi_{T_{j}}\right\|_{2} \\
& =|E|^{\frac{1}{2}}\left(\sum_{j, k}\left|T_{j} \cap T_{k}\right|\right)^{\frac{1}{2}} \\
& \lesssim|E|^{\frac{1}{2}}\left(\sum_{j, k} \frac{\delta^{2}}{\theta\left(e_{j}, e_{k}\right)+\delta}\right)^{\frac{1}{2}}
\end{aligned}
$$

For fixed $k$ the sum over $j$ is $\lesssim \sum_{j:|j-k| \leq \frac{C}{\delta}} \frac{\delta^{2}}{|j-k| \delta+\delta} \lesssim \delta \log \frac{1}{\delta}$. We conclude that $M \lambda \delta \lesssim$ $|E|^{\frac{1}{2}}\left(M \delta \log \frac{1}{\delta}\right)^{\frac{1}{2}}$ which gives the result since $M \gtrsim \frac{|\Omega|}{\delta}$.

Now we show how to pass to the Hausdorff dimension statement. The next result is Lemma 2.15 in [7].

$\underline{\text { Lemma } 1.6}$ Assume an estimate in $\mathbb{R}^{n}$

$$
\left\|f_{\delta}^{*}\right\|_{q, \infty} \leq C \delta^{-\alpha}\|f\|_{p, 1}
$$

Then Kakeya sets have dimension at least $n-p \alpha$.

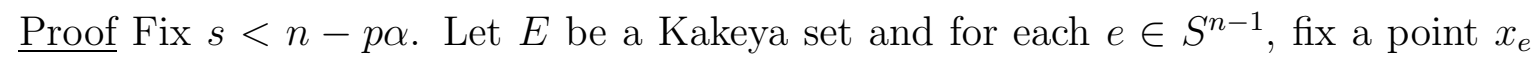
such that $x_{e}+t e \in E$ when $t \in\left[-\frac{1}{2}, \frac{1}{2}\right]$. We have to bound $H_{s}(E)$ from below, so fix a covering of $E$ by discs $D_{j}=D\left(x_{j}, r_{j}\right)$. We can evidently suppose all $r_{j}$ 's are $\leq 1$.

Let $\Sigma_{k}=\left\{j: 2^{-k} \leq r_{j} \leq 2^{-(k-1)}\right\}, \nu_{k}=\left|\Sigma_{k}\right|$ and $E_{k}=E \cap\left(\cup\left\{D_{j}: j \in \Sigma_{k}\right\}\right)$. Also let $\tilde{D}_{j}=D\left(x_{j}, 2 r_{j}\right)$, and $\tilde{E}_{k}=\cup\left\{\tilde{D}_{j}: j \in \Sigma_{k}\right\}$.

Then $\cup_{k} E_{k}=E$, so for each $e$ the pigeonhole principle implies $\mid\left\{t \in\left[-\frac{1}{2}, \frac{1}{2}\right]: x_{e}+t e \in\right.$ $\left.E_{k}\right\} \mid \geq \frac{c}{k^{2}}$ for some $k=k_{e}$, where $c=\frac{6}{\pi^{2}}$. By the pigeonhole principle again, we can find a fixed $k$ so that $k=k_{e}$ when $e \in \Omega$, where $\Omega \subset S^{n-1}$ has measure $\geq \frac{c}{k^{2}}$. With this $k$, we note that $\tilde{E}_{k}$ contains a disc of radius $2^{-k}$ centered at each point of $E_{k}$; it follows easily that if $e \in \Omega$ then $\left|T_{e}^{2^{-k}}\left(x_{e}\right) \cap \tilde{E}_{k}\right| \gtrsim k^{-2}\left|T_{e}^{2^{-k}}\left(x_{e}\right)\right|$. With $f=\chi_{\tilde{E}_{k}}$ we therefore have

$$
\left|\left\{e: f_{2^{-k}}^{*}(e) \geq C^{-1} k^{-2}\right\}\right| \gtrsim k^{-2}
$$

On the other hand, by the assumption (7)

$$
\left|\left\{e: f_{2^{-k}}^{*}(e) \geq C^{-1} k^{-2}\right\}\right| \lesssim\left(k^{2} 2^{k \alpha}\left|\tilde{E}_{k}\right|^{\frac{1}{p}}\right)^{q}
$$

and $\left|\tilde{E}_{k}\right| \lesssim \nu_{k} 2^{-k n}$. So $\left(k^{2} 2^{k \alpha}\left(\nu_{k} 2^{-k n}\right)^{\frac{1}{p}}\right)^{q} \gtrsim k^{-2}$, or equivalently $\nu_{k} \gtrsim k^{-\frac{2}{p}\left(1+\frac{1}{q}\right)} 2^{k(n-p \alpha)}$. Letting $\epsilon=n-p \alpha-s>0$, we have $\sum_{j} r_{j}^{s} \gtrsim \nu_{k} 2^{-k s} \gtrsim k^{-2 p\left(1+\frac{1}{q}\right)} 2^{k \epsilon} \geq$ constant. 
Applying this with $p=n=2$ we see that Proposition 1.5 implies Davies' theorem that Kakeya sets in $\mathbb{R}^{2}$ have dimension 2. Likewise it follows that yes on question 2 for a given $n$ will imply yes on question 1 for the same $n$.

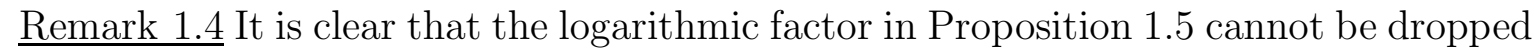
entirely, since then the above argument would show that measure zero Kakeya sets could not exist. In fact it has been known for a long time that the exponent $\frac{1}{2}$ cannot be improved, and U. Keich [29] recently showed that even a higher order improvement is not possible in Proposition 1.5 or in its corollary on $L^{p}$ for $p>2$. On the other hand, a number of related questions concerning logarithmic factors have been solved only recently or are still open. In particular we should mention the results of Barrionuevo [2] and Katz $[26],[27]$ on the question of maximal functions defined using families of directions in the plane.

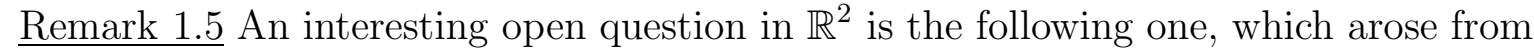
work of Furstenburg.

For a given $\alpha \in(0,1]$, suppose that $E$ is a compact set in the plane, and for each $e \in S^{1}$ there is a line $\ell_{e}$ with direction $e$ such that $\operatorname{dim}\left(\ell_{e} \cap E\right) \geq \alpha$. Then what is the smallest possible value for $\operatorname{dim} E$ ?

Easy results here are that $\operatorname{dim} E \geq \max \left(2 \alpha, \frac{1}{2}+\alpha\right)$ and that there is an example with $\operatorname{dim} E=\frac{1}{2}+\frac{3}{2} \alpha$. We give proofs below. Several people have unpublished results on this question and it is unlikely that the author was the first to observe these bounds; in all probability they are due to Furstenburg and Katznelson.

The analogous discrete question is solved by the following result due to Szemeredi and Trotter [56] (see also [17], [40]).

Suppose we are given $n$ points $\left\{p_{i}\right\}$ and $k$ lines $\left\{\ell_{j}\right\}$ in the plane. Define a line and point to be incident, $p \sim \ell$, if $p$ lies on $\ell$. Let $\mathcal{I}=\left\{(i, j): p_{i} \sim \ell_{j}\right\}$. Then $|\mathcal{I}| \lesssim(k n)^{\frac{2}{3}}+k+n$, and this bound is sharp.

We note that the weaker bound $|\mathcal{I}| \lesssim(k n)^{\frac{3}{4}}+k+n$ follows from $(3)$ and was known long before [56]. To see the analogy with the Hausdorff dimension question, reformulate the Szemeredi-Trotter bound as follows: if each line is incident to at least $\mu$ points $(\mu>>1)$, then (since $|\mathcal{I}| \geq k \mu$ )

$$
n \gtrsim \min \left(\mu^{\frac{3}{2}} k^{\frac{1}{2}}, \mu k\right)
$$

Now assume say ${ }^{2}$ that $E$ has a covering by $n \operatorname{discs} D_{i}$ of radius $\delta$. Consider a set of $k \approx \delta^{-1}$

\footnotetext{
${ }^{2}$ In this heuristic argument we ignore the distinction between Hausdorff and Minkowski dimension.
} 
$\delta$-separated directions $\left\{e_{j}\right\}$. For each $j$ the line $\ell_{e_{j}}$ will intersect $D_{i}$ for at least $\delta^{-\alpha}$ values of $i$. We now pretend that we can replace points by the discs $D_{i}$ in Szemeredi-Trotter and apply (8) with $\mu=\delta^{-\alpha}, k=\delta^{-1}$. Since $k \geq \mu$ we would obtain $n \gtrsim \delta^{-\frac{1}{2}-\frac{3}{2} \alpha}$, i.e. that the bound $\operatorname{dim} E \geq \frac{1}{2}+\frac{3}{2} \alpha$ should hold.

In one situation to be discussed in section 3, it turns out that this kind of heuristic argument can be justified leading to a theorem in the continuum. In other situations such as the present one, it seems entirely unclear whether this should be the case or not, but still the discrete results suggest plausible conjectures.

If correct the bound $\operatorname{dim} E \geq \frac{1}{2}+\frac{3}{2} \alpha$ would be best possible by essentially the same example (due to Erdos, see [40]) that shows the Szemeredi-Trotter bound is sharp.

We start by recalling that if $\left\{n_{j}\right\}$ is a sequence of integers which increases sufficiently rapidly, and if $\alpha \in(0,1)$ then the set

$$
T \stackrel{\text { def }}{=}\left\{x \in\left(\frac{1}{4}, \frac{3}{4}\right): \forall j \exists p, q \in \mathbb{Z}: q \leq n_{j}^{\alpha} \text { and }\left|x-\frac{p}{q}\right| \leq n_{j}^{-2}\right\}
$$

has Hausdorff dimension $\alpha$. This is a version of Jarnik's theorem - see [21], p. 134, Theorem 8.16(b).

It follows that the set

$$
T^{\prime}=\left\{t: \frac{1-t}{t \sqrt{2}} \in T\right\}
$$

also has dimension $\alpha$.

For fixed $n$, consider the set of all line segments $\ell_{j k}$ connecting a point $\left(0, \frac{j}{n}\right)$ to a point $\left(1, \frac{k}{n} \sqrt{2}\right)$, where $j$ and $k$ are any integers between 0 and $n-1$. Thus $\ell_{j k}=\left\{\left(x, \phi_{j k}(x)\right)\right.$ : $0 \leq x \leq 1\}$ where $\phi_{j k}(x)=(1-x) \frac{j}{n}+x \frac{k}{n} \sqrt{2}$. It follows using e.g. [32], p. 124, example 3.2 that every number in $[0,1]$ differs by $\lesssim n^{-2}(\log n)^{2}$ from the slope of one of the $\ell_{j k}$ 's, so the set $G_{n} \stackrel{\text { def }}{=} \cup_{j k} S_{\ell_{j k}}^{n^{-2}(\log n)^{3}}$ is a G-set.

Define

$$
Q_{n}=\left\{t: \frac{1-t}{t \sqrt{2}} \text { is a rational number } \frac{p}{q} \in\left(\frac{1}{4}, \frac{3}{4}\right) \text { with denominator } q \leq n^{\alpha}\right\}
$$

If $t \in Q_{n}$, then let $S(t) \stackrel{\text { def }}{=}\left\{\phi_{j k}(t)\right\}_{j, k=0}^{n-1}$. For any $j$ and $k$ we have $(t \sqrt{2})^{-1} \phi_{j k}(t)=\frac{p j+q k}{q n}$, a rational with denominator $q n$. We conclude that $|S(t)| \lesssim q n \leq n^{1+\alpha}$, hence $\mid \cup(S(t)$ : $\left.t \in Q_{n}\right) \mid \lesssim n^{1+3 \alpha}$ and

(*) The set $\left\{(x, y) \in G_{n}:|x-t| \leq \frac{1}{n^{2}}\right.$ for some $\left.t \in Q_{n}\right\}$ is contained in the union of $\lesssim n^{1+3 \alpha}$ discs of radius $n^{-2}(\log n)^{3}$.

Now we let $\left\{n_{j}\right\}$ increase rapidly and will recursively construct compact sets $F_{j}$ such that $F_{j+1} \subset F_{j}$, each $F_{j}$ is a G-set and the set $\left\{(x, y) \in F_{j}: x \in T^{\prime}\right\}$ is contained in the 
union of $n_{j}^{1+3 \alpha} \log n_{j}$ discs of radius $n_{j}^{-2}\left(\log n_{j}\right)^{3}$. Namely, let $F_{0}$ be any G-set. If $F_{j}$ has been constructed it will be of the form

$$
\cup_{i=1}^{M} S_{\ell_{i}}^{\delta}
$$

for a certain $\delta$, where $\ell_{i}=\left\{\left(x, m_{i} x+b_{i}\right): 0 \leq x \leq 1\right\}$ for suitable $m_{i}$ and $b_{i}$, and every number in $[0,1]$ is within $\delta$ of one of the $m_{i}$. As in the proof of Lemma 1.3 we let $A_{i}(x, y)=\left(x, m_{i} x+\delta y+b_{i}\right)$. We make $n_{j+1}$ sufficiently large and define

$$
F_{j+1}=\cup_{i=1}^{M} A_{i}\left(G_{n_{j+1}}\right)
$$

Clearly $F_{j+1} \subset F_{j}$, and it follows as in Lemma 1.3 that the resulting set is a G-set. The covering property is also essentially obvious from $(*)$ provided $n_{j+1}$ is large enough, say $\log \left(n_{j+1}\right)>>M$.

Let $F=\cap_{j} F_{j}$, and let $E=\left\{(x, y) \in F: x \in T^{\prime}\right\}$. Then the covering property in the construction of $F_{j}$ implies that $\operatorname{dim} E \leq \frac{1}{2}(1+3 \alpha)$. On the other hand $F$ is a G-set, and if $\ell$ is a line segment contained in $F$, then $\operatorname{dim}(\ell \cap E)=\operatorname{dim} T^{\prime} \geq \alpha$. This completes the construction.

We now discuss the bound $\operatorname{dim} E \geq \max \left(2 \alpha, \frac{1}{2}+\alpha\right)$. The bound $\operatorname{dim} E \geq 2 \alpha$ can be derived from Proposition 1.5 by an argument like the proof of Lemma 1.6; we will omit this. To prove the bound $\operatorname{dim} E \geq \frac{1}{2}+\alpha$ (which corresponds to the easy $|\mathcal{I}| \lesssim(k n)^{\frac{3}{4}}+k+n$ under the above heuristic argument) fix a compact set $E$ and for each $e \in S^{1}$ a line $\ell_{e}$ which intersects $E$ in dimension $\geq \alpha$. Let $\left\{D_{j}\right\}=\left\{D\left(x_{j}, r_{j}\right)\right\}$ be a covering. Fix $\beta_{1}<\beta<\alpha$; we have to bound $\sum_{j} r_{j}^{\frac{1}{2}+\beta_{1}}$ from below. As in the proof of Lemma 1.6 we let $\Sigma_{k}=\left\{j: 2^{-k} \leq r_{j} \leq 2^{-(k-1)}\right\}, \nu_{k}=\left|\Sigma_{k}\right|$ and $E_{k}=E \cap\left(\cup\left\{D_{j}: j \in \Sigma_{k}\right\}\right)$. We start by choosing a number $k$ and a subset $\Omega \subset S^{1}$ with measure $\gtrsim \frac{1}{k^{2}}$ such that if $e \in \Omega$ then $H_{\beta}\left(\ell_{e} \cap E_{k}\right) \geq C^{-1} k^{-2}$, using the pigeonhole principle as in the proof of Lemma 1.6. Let $\gamma=\frac{2}{\beta}$. Since $H_{\beta}(I) \leq|I|^{\beta}$ for any interval $I$, it follows that for a suitable numerical constant $C$, and for any $e \in \Omega$ there are two intervals $I_{e}^{ \pm}$on $\ell_{e}$ which are $C^{-1} k^{-\gamma}$ - separated and such that $H_{\beta}\left(E_{k} \cap I_{e}^{ \pm}\right) \gtrsim k^{-2}$. Let $\left\{e_{i}\right\}_{i=1}^{M}$ be a $2^{-k}$-separated subset of $\Omega$ with $M \gtrsim \frac{2^{k}}{k^{2}}$ (see (6)) and define

$$
\mathcal{T}=\left\{\left(j_{+}, j_{-}, i\right) \in \Sigma_{k} \times \Sigma_{k} \times\{1, \ldots, M\}: I_{e_{i}}^{+} \cap E_{k} \cap D_{j_{+}} \neq \emptyset, I_{e_{i}}^{-} \cap E_{k} \cap D_{j_{-}} \neq \emptyset\right\}
$$

We will count $\mathcal{T}$ in two different ways.

First fix $j_{+}$and $j_{-}$and consider how many values of $i$ there can be with $\left(j_{+}, j_{-}, i\right) \in \mathcal{T}$. We will call such a value of $i$ allowable. If the distance between $D_{j_{+}}$and $D_{j_{-}}$is small compared with $k^{-\gamma}$ then there is no allowable $i$, since the distance between $I_{e_{i}}^{+}$and $I_{e_{i}}^{-}$ is always $\geq C^{-1} k^{-\gamma}$. On the other hand if the distance between $D_{j_{+}}$and $D_{j_{-}}$is $\gtrsim k^{-\gamma}$, then because the $\left\{e_{i}\right\}$ are $2^{-k}$-separated, it follows that there are $\lesssim k^{\gamma} i$ 's such that $\ell_{e_{i}}$ 
intersects both $D_{j_{+}}$and $D_{j_{-}}$. Hence in either case there are $\lesssim k^{\gamma}$ allowable $i$ 's. Summing over $j_{+}$and $j_{-}$we conclude that

$$
|\mathcal{T}| \lesssim k^{\gamma} \nu_{k}^{2}
$$

On the other hand, for any fixed $i$, the lower bound $H_{\beta}\left(E_{k} \cap I_{e_{i}}^{+}\right) \gtrsim k^{-2}$ implies there are $\gtrsim k^{-\gamma} 2^{k \beta}$ values of $j_{+}$such that $I_{e_{i}}^{+} \cap E_{k} \cap D_{j_{+}} \neq \emptyset$ and similarly with + replaced by -. So $|\mathcal{T}| \gtrsim M\left(k^{-\gamma} 2^{k \beta}\right)^{2}$. Comparing this bound with (10) we conclude that

$$
\begin{aligned}
\nu_{k} & \gtrsim k^{-\frac{3}{2} \gamma} 2^{k \beta} \sqrt{M} \\
& \gtrsim k^{-\left(1+\frac{3}{2} \gamma\right)} 2^{\left(\frac{1}{2}+\beta\right) k} \\
& \gtrsim 2^{\left(\frac{1}{2}+\beta_{1}\right) k}
\end{aligned}
$$

and therefore $\sum_{j \in \Sigma_{k}} r_{j}^{\frac{1}{2}+\beta_{1}} \geq$ constant.

\section{The higher dimensional case}

We will first make a few remarks about the corresponding problem over finite fields, which is the following:

Let $\mathbb{F}_{q}$ be the field with $q$ elements and let $V$ be an $n$-dimensional vector space over $\mathbb{F}_{q}$. Let $E$ be a subset of $V$ which contains a line in every direction, i.e.

$$
\forall e \in V \backslash\{0\} \exists a \in V: a+t e \in E \forall t \in \mathbb{F}_{q}
$$

Does it follow that $|E| \geq C_{n}^{-1} q^{n}$ ?

Of course $C_{n}$ should be independent of $q$. One could ask instead for a bound like $\forall \epsilon>0 \exists C_{n \epsilon}:|E| \geq C_{n \epsilon}^{-1} q^{n-\epsilon}$ or could restrict to the case of prime fields $\mathbb{F}_{p}$ or fields with bounded degree over the prime field.

So far as I have been able to find out this question has not been considered, and the simple result below corresponds to what is known in the Euclidean case.

$\underline{\text { Proposition } 2.1}$ In the above situation $|E| \geq C_{n}^{-1} q^{\frac{n+2}{2}}$

We give the proof since it is based on the same idea as the $\mathbb{R}^{n}$ proof but involves no technicalities.

First consider the case $n=2$, which is analogous to Proposition 1.5. We will actually prove the following more general statement, which we need below: suppose (with $\operatorname{dim} V=$ 2 ) that $E$ contains at least $\frac{q}{2}$ points on a line in each of $m$ different directions. Then

$$
|E| \gtrsim m q
$$

To prove (11), let $\left\{\ell_{j}\right\}_{j=1}^{m}$ be the lines. Any two distinct $\ell_{j}$ 's intersect in a point. Accordingly 


$$
\begin{aligned}
\frac{1}{2} q m & \leq \sum_{j}\left|E \cap \ell_{j}\right| \\
& \leq|E|^{\frac{1}{2}}\left(\sum_{j k}\left|\ell_{j} \cap \ell_{k}\right|\right)^{\frac{1}{2}} \\
& =|E|^{\frac{1}{2}}(m(m-1+q))^{\frac{1}{2}} \\
& \leq|E|^{\frac{1}{2}}(m q)^{\frac{1}{2}}
\end{aligned}
$$

where we used that $m \leq q+1$. It follows that $|E| \gtrsim m q$. Taking $m=q+1$ we obtain the two dimensional case of Proposition 2.1.

Now assume $n \geq 3$. Then $E$ contains $\frac{q^{n}-1}{q-1} \approx q^{n-1}$ lines $\left\{\ell_{j}\right\}$. Fix a number $\mu$ and define a high multiplicity line to be a line $\ell_{k}$ with the following property: for at least $\frac{q}{2}$ of the $q$ points $x \in \ell_{k}$, the set $\left\{j: x \in \ell_{j}\right\}$ has cardinality at least $\mu+1$. Consider two cases: (i) no high multiplicity line exists (ii) a high multiplicity line exists.

In case (i) we define $\tilde{E}=\left\{x \in E: x\right.$ belongs to $\leq \mu \ell_{j}$ 's $\}$. Then $\tilde{E}$ intersects each $\ell_{j}$ in at least $\frac{q}{2}$ points, by definition of case (i). Each point of $\tilde{E}$ belongs to at most $\mu \ell_{j}$ 's so we may conclude that

$$
\begin{aligned}
|E| & \geq|\tilde{E}| \\
& \geq \mu^{-1} \sum_{j}\left|\tilde{E} \cap \ell_{j}\right| \\
& \geq \mu^{-1} q \cdot q^{n-1}
\end{aligned}
$$

In case (ii), let $\left\{\Pi_{i}\right\}$ be an enumeration of the 2- planes containing $\ell_{k}$. By definition of high multiplicity line there are at least $\frac{\mu q}{2}$ lines $\ell_{j}, j \neq k$, which intersect $\ell_{k}$. Each one of them is contained in a unique $\Pi_{i}$, and contains $q-1$ points of $\Pi_{i}$ which do not lie on $\ell_{k}$. Let $\mathcal{L}_{i}$ be the set of lines $\ell_{j}$ which are contained in a given $\Pi_{i}$. Then by (11) we have $\left|E \cap \Pi_{i} \cap\left(V \backslash \ell_{k}\right)\right| \gtrsim q\left|\mathcal{L}_{i}\right|$. The sets $\Pi_{i} \cap\left(V \backslash \ell_{k}\right)$ are pairwise disjoint so we can sum over $i$ to get $|E| \gtrsim q \sum_{i}\left|\mathcal{L}_{i}\right| \geq \frac{q^{2} \mu}{2}$.

If we take $\mu$ to be roughly $q^{\frac{n-2}{2}}$ we obtain $|E| \gtrsim q^{\frac{n+2}{2}}$ in either case (i) or (ii), hence the result.

Remark 2.1 General finite fields do not always resemble the Euclidean case in this sort of problem. For example, the Szemeredi-Trotter theorem is easily seen to be false (e.g. [5] p. 75). A counterexample involving one line in each direction as in remark 1.5 may be obtained in the following way: let $q=p^{2}$ with $p$ prime, let $\alpha$ be a generator of $\mathbb{F}_{q}$ over $\mathbb{F}_{p}$ and in the two dimensional vector space $V$ over $\mathbb{F}_{q}$, let $\ell_{j k}$ be the line connecting $(0, j)$ to $(1, k \alpha)$. Here $j$ and $k$ are in $\mathbb{F}_{p}$. This is a set of $p^{2}$ lines containing one line in each 
direction other than the vertical. For given $t \in \mathbb{F}_{q}$, let $S_{t}=\left\{y \in \mathbb{F}_{q}:(t, y) \in \cup_{j k} \ell_{j k}\right\}$. If $t$ is such that $\alpha \frac{t}{1-t} \in \mathbb{F}_{p}$ then it is easily seen that $S_{t}$ coincides with $(1-t) \mathcal{F}_{p}$, and if $t=1$ then $S_{t}=\alpha \mathbb{F}_{p}$. This gives $p$ "bad" values of $t$ such that $S_{t}$ has cardinality $p$. Let $E=\cup_{t}\left\{(t, y): y \in S_{t}\right\}$, where the union is taken over the bad values of $t$. Then $\left\{\ell_{j k}\right\}$ and $E$ give a configuration of $p^{2}$ lines and $p^{2}$ points with $p^{3}$ incidences, matching the trivial upper bound from (3).

In the $\mathbb{R}^{n}$ context, arguments like the proof of Proposition 2.1 can still be used, except that one has to work with tubes instead of lines and measure instead of cardinality, and take into account such issues as that the size of the intersection of two tubes will depend on the angle of intersection via (4). This was perhaps first done by Cordoba (e.g. [18] see the proof of Proposition 1.5 above). We will present here the "bush" argument from [7], p. 153-4 which shows the following:

$$
\text { Proposition } 2.2\left\|f_{\delta}^{*}\right\|_{n+1, \infty} \leq C_{n} \delta^{-\frac{n-1}{n+1}}\|f\|_{\frac{n+1}{2}, 1}
$$

Proof Using (6), we see that what must be shown is the following: if $\left\{T_{e_{j}}^{\delta}\right\}_{j=1}^{M}$ are tubes with $\delta$-separated directions, $E$ is a set and $\left|E \cap T_{e_{j}}\right| \geq \lambda\left|T_{e_{j}}^{\delta}\right|$, then

$$
|E| \gtrsim \delta^{\frac{n-1}{2}} \lambda^{\frac{n+1}{2}} \sqrt{M}
$$

To this end we fix a number $\mu$ ("multiplicity") and consider the following two possibilities

(i) (low multiplicity) No point of $E$ belongs to more than $\mu$ tubes $T_{e_{j}}^{\delta}$.

(ii) (high multiplicity) Some point $a \in E$ belongs to more than $\mu$ tubes $T_{e_{j}}^{\delta}$.

In case (i) it is clear that $|E| \gtrsim \mu^{-1} \sum_{j}\left|E \cap T_{e_{j}}^{\delta}\right|$, hence

$$
|E| \gtrsim \mu^{-1} M \lambda \delta^{n-1}
$$

In case (ii) we fix a point $a$ as indicated and may assume that $a$ belongs to $T_{e_{j}}^{\delta}$ when $j \leq \mu+1$. If $C_{0}$ is a suitably large fixed constant, then $\left|T_{e_{j}}^{\delta} \cap D\left(a, C_{0}^{-1} \lambda\right)\right| \leq \frac{\lambda}{2}\left|T_{e_{j}}^{\delta}\right|$. Accordingly, for $j \leq \mu+1$, we have

$$
\left|E \cap T_{e_{j}}^{\delta} \cap D\left(a, C_{0}^{-1} \lambda\right)^{c}\right| \geq \frac{\lambda}{2}\left|T_{e_{j}}^{\delta}\right| \gtrsim \lambda \delta^{n-1}
$$

If $j, k \leq \mu$ then $T_{e_{j}}^{\delta} \cap T_{e_{k}}^{\delta}$ contains $a$ and has diameter $\lesssim \frac{\delta}{\theta\left(e_{j}, e_{k}\right)}$ by (4). It follows that if $\theta\left(e_{j}, e_{k}\right) \geq C_{1} \frac{\delta}{\lambda}$ for a suitably large $C_{1}$, then the sets $E \cap T_{e_{j}}^{\delta} \cap D\left(a, C_{0}^{-1} \lambda\right)^{c}$ and 
$E \cap T_{e_{k}}^{\delta} \cap D\left(a, C_{0}^{-1} \lambda\right)^{c}$ are disjoint. We conclude that

$$
|E| \gtrsim \mathcal{N} \cdot \lambda \delta^{n-1}
$$

where $\mathcal{N}$ is the maximum possible cardinality for a $C_{1} \frac{\delta}{\lambda}$-separated subset of $\left\{e_{j}\right\}_{j=1}^{\mu+1}$. Since the $\left\{e_{j}\right\}$ are $\delta$ - separated, we have $\mathcal{N} \gtrsim \lambda^{n-1} \mu$ and therefore

$$
|E| \gtrsim \lambda^{n} \delta^{n-1} \mu
$$

We conclude that for any given $\mu$ either (13) or (14) must hold. Taking $\mu \approx \lambda^{-\left(\frac{n-1}{2}\right)} \sqrt{M}$ we get $(12)$.

Further remarks: 2.2. Bourgain [7] also gave an additional argument leading to an improved result which implies $\operatorname{dim}$ (Kakeya) $\geq \frac{n+1}{2}+\epsilon_{n}$, where $\epsilon_{n}$ is given by a certain inductive formula (in particular $\epsilon_{3}=\frac{1}{3}$ ). A more efficient argument was then given by the author [60], based on considering families of tubes which intersect a line instead of a point as in the bush argument; this is the continuum analogue of the proof of Proposition 2.1. It gives the following bound:

$$
\forall \epsilon \exists C_{\epsilon}:\left\|f_{\delta}^{*}\right\|_{q} \leq C_{\epsilon} \delta^{-\left(\frac{n}{p}-1\right)-\epsilon}\|f\|_{p}
$$

where $p=\frac{n+2}{2}$ and $q=(n-1) p^{\prime}$. This is the estimate on $L^{p}$ which would follow by interpolation with the trivial $\left\|f_{\delta}^{*}\right\|_{\infty} \lesssim \delta^{-(n-1)}\|f\|_{1}$ if the bound (2) could be proved. In particular, it implies the dimension of Kakeya sets is $\geq \frac{n+2}{2}$. Other proofs of estimates like (15) have also recently been given by Katz [28] and Schlag [45]. However in every dimension $n \geq 3$ it is unknown whether (15) holds for any $p>\frac{n+2}{2}$ and whether $\operatorname{dim}($ Kakeya $)>\frac{n+2}{2}$.

2.3. Proposition 2.2 is also a corollary of the $L^{\frac{n+1}{2}} \rightarrow L^{n+1}$ estimate for the x-ray transform due to Drury and Christ [20],[18] (see also [39], [16] for related results). Conversely, a refinement of the argument which proves (15) can be used to prove the estimate on $L^{\frac{n+2}{2}}$ which would follow from (2) and the result of [20] by interpolation, at least in the three dimensional case. See [62].

2.4. We briefly discuss some other related problems. The classical problem of Nikodym sets has been shown to be formally equivalent to the Kakeya problem by Tao [58]; we refer to his paper for further discussion. Another classical problem is the problem of $(n, 2)$ sets: suppose that $E$ is a set in $\mathbb{R}^{n}$ which contains a translate of every 2-plane. Does it follow that $E$ has positive measure? At present this is known only when $n=3$ [33] or $n=4$ [7]. The argument in [7], section 4 shows the following: suppose that (2) can be proved in dimension $n-1$, or more precisely that a slightly weaker result can be proved, namely that for some $p$ and $q$ there is an estimate

$$
\left\|f_{\delta}^{*}\right\|_{L^{q}\left(S^{n-2}\right)} \lesssim \delta^{-\alpha}\|f\|_{L^{p}\left(\mathbb{R}^{n-1}\right)} \text { with } \alpha<\frac{1}{p}
$$


Then $(n, 2)$-sets have positive measure.

However, note that (16) would imply by Lemma 1.6 that Kakeya sets in $\mathbb{R}^{n-1}$ have dimension $\geq n-2$. In fact if an estimate (16) is true for every $n$ then one could answer question 1 affirmatively by an argument based on the fact that the direct product of Kakeya sets is Kakeya. It may therefore be unlikely that the $(n, 2)$-sets problem can be solved without a full understanding of the Kakeya problem. However, the most recent results on it are those of [1].

2.5. If one considers curves instead of lines, then it is known that much less can be expected to be true. This first results in this direction are in [8]; see also [10], [35] and [49].

Added in proof Bourgain recently improved on the results discussed here in sufficiently high dimensions, by showing that the dimension of a Kakeya set in $\mathbb{R}^{n}$ is greater than $\alpha$ n for suitable explicit $\alpha>\frac{1}{2}$. We refer to his forthcoming paper for the details.

\section{Circles}

In this section we will discuss some analogous problems about circles in the plane, or (essentially equivalent) fine estimates for the wave equation in $2+1$ dimensions. These problems are much better understood than the Kakeya problem and yet they present some of the same difficulties.

A prototype result due to Bourgain [6] and Marstrand [34] independently is that

$(*)$ : A set in $\mathbb{R}^{2}$ which contains circles with arbitrary centers must have positive measure.

Bourgain proved a stronger result which has the same relation to $(*)$ as question 2 does to question 1. Namely, define a maximal function

$$
\mathcal{M} f(x)=\sup _{r} \int\left|f\left(x+r e^{i \theta}\right)\right| \frac{d \theta}{2 \pi}
$$

Then $\|\mathcal{M} f\|_{L^{p}\left(\mathbb{R}^{2}\right)} \lesssim\|f\|_{L^{p}\left(\mathbb{R}^{2}\right)}, p>2$. As is well-known, this maximal function was introduced by Stein [51] and he proved the analogous inequality in dimensions $n \geq$ 3 ; the range of $p$ is then $p>\frac{n}{n-1}$. Stein's proof was based partly on the Plancherel theorem and Bourgain's argument in the two dimensional case also used the Plancherel theorem, whereas Marstrand's argument was purely geometric. We will discuss some further developments of the latter approach.

A variant on the Kakeya construction due to Besicovitch-Rado [4] and Kinney [30] shows the following:

(**) There are compact sets in the plane with measure zero containing circles of every radius between 1 and 2 . 
We will call such sets BRK sets. The distinction between $(*)$ and $(* *)$ can be understood in terms of parameter counting: a set as in $(* *)$ is a subset of a 2-dimensional space containing a 1-parameter family of 1-dimensional objects, so whether it has positive measure or not can be expected to be a borderline question. This is analogous to the question of Kakeya sets which also contain $n-1$-parameter families of 1-dimensional objects. On the other hand a set as in $(*)$ contains a 2 - parameter family of 1-dimensional objects in a 2-dimensional space.

A further related remark is that analogous constructions with other 1-parameter families of circles have been done by Talagrand [57]. For example, he shows that for any smooth curve $\gamma$ there are sets of measure zero containing circles centered at all points of $\gamma$.

It is natural to ask whether the dimension of a BRK set must be 2 or not. This question also has a maximal function version; the relevant maximal function is the following $M_{\delta}$ : if $f: \mathbb{R}^{2} \rightarrow \mathbb{R}$ then $M_{\delta} f:\left[\frac{1}{2}, 2\right] \rightarrow \mathbb{R}$,

$$
M_{\delta} f(r)=\sup _{x} \frac{1}{\left|C_{\delta}(x, r)\right|} \int_{C_{\delta}^{(x, r)}}|f|
$$

One shows analogously to Lemma 1.6 that a bound (for some $p<\infty$ )

$$
\forall \epsilon \exists \mathcal{C}_{\epsilon}:\left\|M_{\delta} f\right\|_{L^{p}\left(\left[\frac{1}{2}, 2\right]\right)} \leq C_{\epsilon} \delta^{-\epsilon}\|f\|_{p}
$$

will imply that BRK sets have dimension 2. Note that existence of measure zero BRK sets implies the $\delta^{-\epsilon}$ factor is needed. This is similar to the situation with the two dimensional Kakeya problem. However in contrast to the latter problem it is not possible to take $p=2$ in (18). In fact $p$ must be at least 3 ; this is seen by considering the standard example $f=$ indicator function of a rectangle with dimensions $\delta \times \sqrt{\delta}$.

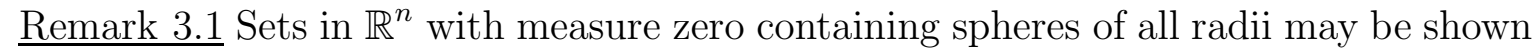
to exist for $n \geq 3$ also, and the maximal function (17) may be defined in $\mathbb{R}^{n}$. However, in that case the questions mentioned above are essentially trivial, since the correct estimate for the maximal function is an $L^{2} \rightarrow L^{2}$ estimate, is easy and implies that sets containing spheres with all radii have dimension $n$. Namely, the estimate

$$
\left\|M_{\delta} f\right\|_{2} \lesssim\left(\log \frac{1}{\delta}\right)^{\frac{1}{2}}\|f\|_{2}
$$

can be proved analogously to Proposition 1.5 and is also closely related to some of the Strichartz inequalities for the wave equation (cf. [41]), due to the fact that spherical means correspond roughly to solutions of the initial value problem $\square u=0, u(\cdot, 0)=$ $f, \frac{\partial u}{\partial t}(\cdot, 0)=0$ after taking $\frac{n-1}{2}$ derivatives. These remarks are from [31]. From a certain 
point of view, the "reason" why the higher dimensional case is easier is the following: if $|r-s| \approx 1$ then

$$
\left|C_{\delta}(x, r) \cap C_{\delta}(y, s)\right| \approx \begin{cases}\delta^{\frac{n+1}{2}} & \text { if } C(x, r) \text { and } C(y, s) \text { are tangent } \\ \delta^{2} & \text { if } C(x, r) \text { and } C(y, s) \text { are sufficiently transverse }\end{cases}
$$

making the first possibility "worse" than the second in $\mathbb{R}^{2}$ but not in higher dimensions.

We now consider only the two dimensional case and will formulate a discrete analogy like the analogy between the Szemeredi-Trotter theorem and the question mentioned in Remark 1.5. The relevant problem in discrete geometry is

Given $N$ circles $\left\{C_{i}\right\}$ in the plane, no three tangent at a point, how many pairs $(i, j)$ can there be such that $C_{i}$ is tangent to $C_{j}$ ?

For technical reasons we always interpret "tangent" as meaning "internally tangent", i.e. a circle $C(x, r)$ is "tangent" to $C(y, s)$, written $C(x, r)|| C(y, s)$, iff $|x-y|=|r-s|$.

We will call this the tangency counting problem. We're not aware of any literature specifically about this problem, but known techniques in incidence geometry (related to the Szemeredi-Trotter theorem) can be adapted to it without difficulty. One obtains the following bounds for $\mathcal{I} \stackrel{\text { def }}{=}\left\{(i, j): C_{i} \| C_{j}\right\}$.

(i) (easy) $|\mathcal{I}| \lesssim N^{\frac{5}{3}}$. This follows from the fact that the incidence matrix

$$
a_{i j}= \begin{cases}1 & \text { if } C_{i} \| C_{j} \\ 0 & \text { otherwise }\end{cases}
$$

contains no $3 \times 3$ submatrix of 1's (essentially a theorem of Appolonius: there are at most two circles which are internally tangent to three given circles at distinct points) and therefore contains at most $\mathcal{O}\left(N^{\frac{5}{3}}\right) 1$ 's by (3).

(ii) (more sophisticated) $\forall \epsilon>0 \exists C_{\epsilon}<\infty:|\mathcal{I}| \lesssim N^{\frac{3}{2}+\epsilon}$. This follows readily from the techniques of Clarkson, Edelsbrunner, Guibas, Sharir and Welzl [17]. We will not discuss their work here; we just note that they prove the analogous $N^{\frac{3}{2}+\epsilon}$ bound in the three dimensional unit distance problem: in our notation, given $\left\{\left(x_{i}, r_{i}\right)\right\}_{i=1}^{N} \subset \mathbb{R}^{2} \times \mathbb{R}$, there are $\lesssim N^{\frac{3}{2}+\epsilon}$ pairs $(i, j)$ with $\left|x_{i}-x_{j}\right|^{2}+\left(r_{i}-r_{j}\right)^{2}=1$.

There is no reason to think that the bound (ii) should be sharp. ${ }^{3}$ However, (ii) leads

\footnotetext{
${ }^{3}$ It may be more natural to consider a slightly different formulation of the problem: drop the assumption that no three circles are tangent at a point, and consider the number of points where two are tangent instead of the number of tangencies. With this reformulation, a standard example involving circles with integer center and radius shows that the exponent $\frac{4}{3}$ would be best possible as in the unit distance problem.
} 
to a sharp result on the BRK sets problem and a proof of the maximal inequality (18) with $p=3$. The heuristic argument is the following: assume we know a bound $\lesssim N^{\alpha}$ in the tangency counting problem, where $\alpha \geq \frac{3}{2}$. Let $E$ be a BRK set and consider its $\delta$-neighborhood $E^{\delta}$. Let $\left\{r_{j}\right\}_{j=1}^{M}$ be a maximal $\delta$-separated subset of $\left[\frac{1}{2}, 2\right]$; then $M \approx \frac{1}{\delta}$ and $E^{\delta}$ contains an annulus $C_{\delta}\left(x_{j}, r_{j}\right)$ for each $j$. By (20), we should have to a first approximation $\left|C_{\delta}\left(x_{j}, r_{j}\right) \cap C_{\delta}\left(x_{k}, r_{k}\right)\right| \approx \delta^{\frac{3}{2}}$ if $C\left(x_{j}, r_{j}\right)$ and $C\left(x_{i}, r_{i}\right)$ intersect tangentially and $\left|C_{\delta}\left(x_{j}, r_{j}\right) \cap C_{\delta}\left(x_{k}, r_{k}\right)\right| \approx \delta^{2}$ if they intersect transversally. Accordingly we would get $\sum_{j k}\left|C_{\delta}\left(x_{j}, r_{j}\right) \cap C_{\delta}\left(x_{k}, r_{k}\right)\right| \lesssim \delta^{-\alpha} \cdot \delta^{\frac{3}{2}}+\delta^{-2} \cdot \delta^{2} \lesssim \delta^{\frac{3}{2}-\alpha}$, and then the argument in the proof of Proposition 1.5 shows that $\left|E_{\delta}\right| \gtrsim \delta^{\frac{1}{2}\left(\alpha-\frac{3}{2}\right)}$, so one expects $\operatorname{dim} E \geq 2-\frac{1}{2}\left(\alpha-\frac{3}{2}\right)$.

It turns out that it is possible to make this argument rigorous and to obtain a corresponding result ((18) with $p=3)$ for the maximal operator. The first lemma below keeps track of the intersection of two annuli in terms of their degree of tangency; it is of course quite standard and is used in one form or another in most papers in the area, e.g. [6] and [34]. The second lemma is due to Marstrand ([34], Lemma 5.2), although he formulated it slightly differently. It gives a quantitative meaning to the theorem of Appolonius used in the proof of the $N^{\frac{5}{3}}$ tangency bound.

We introduce the following notation: if $C(x, r)$ and $C(y, s)$ are circles then

$$
\begin{gathered}
d((x, r),(y, s))=|x-y|+|r-s| \\
\Delta((x, r),(y, s))=|| x-y|-| r-s||
\end{gathered}
$$

Note that $\Delta$ vanishes precisely when the circles are "tangent." In Lemmas 3.1 and 3.2 below, we assume that all circles $C(x, r)$ etc. have centers in $D\left(0, \frac{1}{4}\right)$ and radii between $\frac{1}{2}$ and 2 .

Lemma 3.1 Assume that $x \neq y$. Let $d=d((x, r),(y, s)), \Delta=\Delta((x, r),(y, s))$, and $e=\operatorname{sgn}(r-s) \frac{y-x}{|y-x|}, \zeta=y+r e$. Then

(a) $C_{\delta}(x, r) \cap C_{\delta}(y, s)$ is of measure $\lesssim \delta \cdot \frac{\delta}{\sqrt{(\delta+\Delta)(\delta+d)}}$.

(b) $C_{\delta}(x, r) \cap C_{\delta}(y, s)$ is contained in a disc centered at $\zeta$ with radius $\lesssim \sqrt{\frac{\Delta+\delta}{d+\delta}}$.

Proof We use the following fact: if $\mu>0, \epsilon>0$ then the set $\{x \in[-\pi, \pi]:|\cos x-\mu| \leq$ $\epsilon\}$ is (i) contained in the union of two intervals of length $\lesssim \frac{\epsilon}{\sqrt{|1-\mu|}}$ and (ii) contained in an interval of length $\lesssim \sqrt{|1-\mu|+\epsilon}$ centered at 0 .

To prove the lemma, we use complex notation and may assume that $x=0, r=1, y$ is on the positive real axis and $s<1$. Note that then $e=1$. If $d \leq 4 \delta$ then the lemma is 
trivial, and if $y<\frac{d}{2}-\delta$ then $y+s<1-2 \delta$ so that $C_{\delta}(0,1) \cap C_{\delta}(y, s)=\emptyset$. So we can assume that $d \geq 4 \delta$ and $y \geq \frac{d}{2}-\delta \geq \frac{d}{4}$.

If $z \in C_{\delta}(0,1) \cap C_{\delta}(y, s)$ then clearly $\left|z-e^{i \theta}\right| \leq \delta$ for some $\theta \in[-\pi, \pi]$. It suffices to show that the set of $\theta$ which can occur here is contained in two intervals of length $\lesssim \frac{\delta}{\sqrt{(\delta+\Delta)(\delta+d)}}$ and in an interval of length $\lesssim \sqrt{\frac{\Delta+\delta}{d+\delta}}$ centered at 0 .

The point $e^{i \theta}$ must belong to $C_{2}(y, s)$, i.e. ||$e^{i \theta}-y|-s|<2 \delta$ and therefore, since ||$e^{i \theta}-y|+s| \approx 1$

$$
|| e^{i \theta}-\left.y\right|^{2}-s^{2} \mid \lesssim \delta
$$

We can express this as

$$
\left|\cos \theta-\frac{1+y^{2}-s^{2}}{2 y}\right| \lesssim \frac{\delta}{y} \lesssim \frac{\delta}{d}
$$

Let $\mu=\frac{1+y^{2}-s^{2}}{2 y}, \epsilon=C \frac{\delta}{d}$. Then $\mu$ is positive, and $|1-\mu|=\frac{\left|s^{2}-(1-y)^{2}\right|}{2 y} \approx \frac{|1-s-y|}{2 y} \approx \frac{\Delta}{d}$. Apply fact (ii) in the first paragraph. The set of possible $\theta$ is therefore contained in an interval of length $\lesssim \sqrt{\frac{\Delta+\delta}{d}}$ centered at 0 . This proves (b), since we are assuming $d \geq \delta$. Estimate (a) follows from (b) if $\Delta \leq \delta$. If $\Delta \geq \delta$, then fact (i) in the first paragraph gives the additional property that $\theta$ must be contained in the union of two intervals of length $\lesssim \frac{\delta / d}{\sqrt{\Delta / d}} \approx \frac{\delta}{\sqrt{(\delta+\Delta)(\delta+d)}}$

Lemma 3.2 (Marstrand's 3-circle lemma) For a suitable numerical constant $C_{0}$, assume that $\epsilon, t, \lambda \in(0,1)$ satisfy $C_{0} \frac{\epsilon}{t} \leq \lambda^{2}$. Fix three circles $C\left(x_{i}, r_{i}\right), 1 \leq i \leq 3$. Then for $\delta \leq \epsilon$ the set

$$
\begin{aligned}
\bar{\Omega}_{\epsilon t \lambda \stackrel{\text { def }}{=}} & \left\{(x, r) \in \mathbb{R}^{2} \times \mathbb{R}: \Delta\left((x, r),\left(x_{i}, r_{i}\right)\right)<\epsilon \forall i,\right. \\
& d\left((x, r),\left(x_{i}, r_{i}\right)\right)>t \forall i, C_{\delta}(x, r) \cap C_{\delta}\left(x_{i}, r_{i}\right) \neq \emptyset \forall i, \\
& \left.\operatorname{dist}\left(C_{\delta}(x, r) \cap C_{\delta}\left(x_{i}, r_{i}\right), C_{\delta}(x, r) \cap C_{\delta}\left(x_{j}, r_{j}\right)\right) \geq \lambda \forall i, j: i \neq j\right\}
\end{aligned}
$$

is contained in the union of two ellipsoids in $\mathbb{R}^{3}$ each of diameter $\lesssim \frac{\epsilon}{\lambda^{2}}$ and volume $\lesssim \frac{\epsilon^{3}}{\lambda^{3}}$.

Proof This will be based on the inverse function theorem. We remark that the sketch of proof given in [61] is inaccurate.

We will actually work with a slightly different set, namely, with

$$
\begin{aligned}
\Omega_{\epsilon t \lambda}= & \left\{(x, r) \in \mathbb{R}^{2} \times \mathbb{R}: \Delta\left((x, r),\left(x_{i}, r_{i}\right)\right)<\epsilon \forall i, d\left((x, r),\left(x_{i}, r_{i}\right)\right)>t \forall i,\right. \\
& \left.\left|e_{i}(x, r)-e_{j}(x, r)\right| \geq \lambda \forall i, j: i \neq j\right\}
\end{aligned}
$$

where $e_{i}(x, r)=\operatorname{sgn}\left(r-r_{i}\right) \frac{x_{i}-x}{\left|x_{i}-x\right|}$. This is sufficient since by Lemma $3.1(\mathrm{~b}), \Omega_{\epsilon t \frac{\lambda}{2}}$ will contain $\bar{\Omega}_{\epsilon t \lambda}$ provided $C_{0}$ is sufficiently large. 
If $e_{1}, \ldots, e_{4}$ are unit vectors in $\mathbb{R}^{2}$ which are contained in an arc of length $\mu$, then the reader will convince herself or himself that

$$
\left|\left(e_{1}-e_{2}\right) \wedge\left(e_{3}-e_{4}\right)\right| \lesssim \mu\left|e_{1}-e_{2}\right|\left|e_{3}-e_{4}\right|
$$

and furthermore if $e_{1}, e_{2}, e_{3}$ are unit vectors in $\mathbb{R}^{2}$ then

$$
\left|\left(e_{1}-e_{2}\right) \wedge\left(e_{1}-e_{3}\right)\right| \approx\left|e_{1}-e_{2}\right|\left|e_{2}-e_{3}\right|\left|e_{3}-e_{1}\right|
$$

Here $\wedge$ is wedge product, $(a, b) \wedge(c, d)=a d-b c$.

Consider the map $G: \mathbb{R}^{2} \times \mathbb{R} \rightarrow \mathbb{R}^{3}$ defined by

$$
G(x, \rho)=\left(\begin{array}{c}
\left|x-x_{1}\right|-\left|r-r_{1}\right| \\
\left|x-x_{2}\right|-\left|r-r_{2}\right| \\
\left|x-x_{3}\right|-\left|r-r_{3}\right|
\end{array}\right)
$$

$\operatorname{Fix}(\xi, \rho) \in \Omega_{\epsilon t \lambda}$. Observe that

$$
D G(\xi, \rho) \simeq\left(\begin{array}{ll}
e_{1}(\xi, \rho) & -1 \\
e_{2}(\xi, \rho) & -1 \\
e_{3}(\xi, \rho) & -1
\end{array}\right)
$$

where " $\simeq$ " means that the two matrices are equal after each row of the matrix on the right hand side is multiplied by an appropriate choice of \pm 1 .

We can assume that $\left|e_{1}(\xi, \rho)-e_{3}(\xi, \rho)\right| \geq\left|e_{1}(\xi, \rho)-e_{2}(\xi, \rho)\right| \geq\left|e_{2}(\xi, \rho)-e_{3}(\xi, \rho)\right|$. Let $\mu=\left|e_{1}(\xi, \rho)-e_{3}(\xi, \rho)\right|, \nu=\left|e_{2}(\xi, \rho)-e_{3}(\xi, \rho)\right|$; then we have $\mu \geq \nu \gtrsim \lambda$ and also $\left|e_{1}(\xi, \rho)-e_{2}(\xi, \rho)\right| \approx \mu$. It follows by $(22)$ that $|\operatorname{det} D G(\xi, \rho)| \approx \mu^{2} \nu$. Furthermore, all entries in the cofactor matrix of $D G(\xi, \rho)$ are easily seen to be $\lesssim \mu$. Let $E(\xi, \rho)=$ $\left\{(x, r) \in \mathbb{R}^{2} \times \mathbb{R}:|D G(\xi, \rho)(x-\xi, r-\rho)|<A \epsilon\right\}$ for an appropriate large constant $A$ which should be chosen before $C_{0}$. Then the preceding considerations imply $E(\xi, \rho)$ is an ellipsoid with

$$
\begin{gathered}
\operatorname{diam}(E(\xi, \rho)) \lesssim \frac{\epsilon}{\mu \nu} \\
|E(\xi, \rho)| \lesssim \frac{\epsilon^{3}}{\mu^{2} \nu}
\end{gathered}
$$

We claim that if $(x, r) \in E$ then $D G(x, r) D G(\xi, \rho)^{-1}=I+E$, where $I$ is the $3 \times 3$ identity matrix and $E$ is a matrix with norm $\leq \frac{1}{100}$, say.

A matrix calculation shows that each entry of $(D G(x, r)-D G(\xi, \rho)) D G(\xi, \rho)^{-1}$ has the form $(\operatorname{det} D G(\xi, \rho))^{-1}\left(e_{i}(x, r)-e_{i}(\xi, \rho)\right) \wedge\left(e_{j}(\xi, \rho)-e_{k}(\xi, \rho)\right)$ for appropriate $i, j, k$. We will show below that

$$
\left|e_{i}(x, r)-e_{i}(\xi, \rho)\right| \lesssim \frac{\epsilon}{t \nu}
$$


If we assume this then the claim may be proved as follows. (26) implies in particular that all the vectors $e_{i}(x, r)$ and $e_{j}(\xi, \rho)$ belong to an arc of length $\lesssim \mu$. Accordingly, using $(21)$,

$$
\begin{aligned}
& \left|\operatorname{det} D G(\xi, \rho)^{-1}\left(e_{i}(x, r)-e_{i}(\xi, \rho)\right) \wedge\left(e_{j}(\xi, \rho)-e_{k}(\xi, \rho)\right)\right| \\
\lesssim & \mu\left|\operatorname{det} D G(\xi, \rho)^{-1}\right|\left|e_{i}(x, r)-e_{i}(\xi, \rho)\right|\left|e_{j}(\xi, \rho)-e_{k}(\xi, \rho)\right| \\
\lesssim & \mu \cdot\left(\mu^{2} \nu\right)^{-1} \cdot \frac{\epsilon}{t \nu} \cdot \mu \\
\leq & \frac{\epsilon}{t \nu^{2}}
\end{aligned}
$$

which is small.

To prove (26) we abbreviate $e_{i}=e_{i}(\xi, \rho)$. Fix $i$ and let $e_{i}^{*} \in \mathbb{R}^{2}$ be a unit vector perpendicular to $e_{i}$. If we define $j$ and $k$ via $\{i, j, k\}=\{1,2,3\}$, then a little linear algebra shows that $e_{i}^{*}=\alpha\left(e_{i}-e_{j}\right)+\beta\left(e_{i}-e_{k}\right)$ with $|\alpha|+|\beta| \lesssim \nu^{-1}$. Furthermore, if we let $\left(v_{1}, v_{2}, v_{3}\right)=D G(\xi, \rho)(x-\xi, r-\rho)$, then by $(23)$ we have $\left|\left(e_{i}-e_{j}\right) \cdot(x-\xi)\right|=\left|v_{i} \pm v_{j}\right| \leq 2 \epsilon$ and similarly $\left|\left(e_{i}-e_{k}\right) \cdot(x-\xi)\right| \leq 2 \epsilon$. We conclude that $\left|e_{i}^{*} \cdot(x-\xi)\right| \lesssim \frac{\epsilon}{\nu}$, hence $\left|e_{i}^{*} \cdot\left(x-x_{i}\right)\right| \lesssim \frac{\epsilon}{\nu}$ since $x_{i}-\xi$ is parallel to $e_{i}$. Also $\left|x-x_{i}\right| \geq \frac{t}{2}$ by $(24)$, so

$$
\left|e_{i}^{*} \cdot \frac{x-x_{i}}{\left|x-x_{i}\right|}\right| \lesssim \frac{\epsilon}{t \nu}
$$

This implies that for an appropriate choice of \pm

$$
\left|e_{i}(x, r) \pm e_{i}\right| \lesssim \frac{\epsilon}{t \nu}
$$

Note though that $r-r_{i}$ and $\left|x-x_{i}\right|$ are nonzero on $E(\xi, \rho)$ : this follows from (24), since $\epsilon$ is small compared with $t$ so that $\left|\xi-x_{i}\right| \approx t \approx\left|\rho-r_{i}\right|$. So $(x, r) \rightarrow e_{i}(x, r)$ is a continuous function on $E(\xi, \rho)$ and therefore the sign in (27) is independent of $(x, r)$. So (26) holds and the claim is proved.

If $A$ is large enough then the claim implies via the usual proof of the inverse function theorem that $G$ is a diffeomorphism from a subset of $E(\xi, \rho)$ onto a disc of radius $2 \epsilon$, say. In particular, $E(\xi, \rho)$ must contain a point $(x, r)$ with $G(x, r)=0$. Then $C(x, r)$ is internally tangent to each $C\left(x_{i}, r_{i}\right)$; note that by (26) and the bound on the diameter of $E$, we have $(x, r) \in \Omega_{\epsilon \frac{t}{2} \frac{\lambda}{2}}$ and furthermore, by the claim $E(x, r)$ and $E(\xi, \rho)$ are comparable ellipsoids (each is contained in a fixed dilate of the other). Appolonius' theorem implies there are only two possibilities for the circle $C(x, r)$, and we have just seen that $(\xi, \rho)$ must be contained in one of the two $E(x, r)$ 's and that they have the proper dimensions.

$\underline{\text { Proposition } 3.3}$ For any $p<\frac{8}{3}$ there is an estimate

$$
\left\|M_{\delta} f\right\|_{q} \leq C \delta^{-\frac{1}{2}\left(\frac{3}{p}-1\right)}\|f\|_{p}, q=2 p^{\prime}
$$


This implies by the proof of Lemma 1.6 that BRK sets have dimension $\geq 2-\frac{1}{2}\left(\frac{3}{p}-1\right)$ for any $p<\frac{8}{3}$, i.e. dimension $\geq \frac{11}{6}$. Proposition 3.3 was proved (in generalized form) in [31]; it is the partial result which corresponds to the bound (i) in the tangency counting problem. The sharp result $((18)$ with $p=3)$ incorporating the technique from [17] is proved in [61].

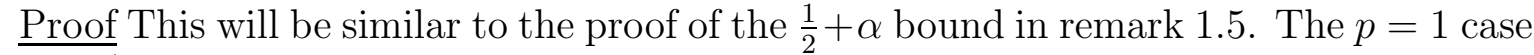
is trivial ${ }^{4}$ so it suffices to prove the following restricted weak type bound at the endpoint:

$$
\left|\left\{r \in\left[\frac{1}{2}, 2\right]: M_{\delta} \chi_{E}(r)>\lambda\right\}\right| \leq C\left(\frac{|E|}{\delta^{\frac{1}{6}} \lambda^{\frac{8}{3}}}\right)^{\frac{6}{5}}
$$

We may assume in proving (28) that the diameter of the set $E$ is less than one. Consequently in defining $M_{\delta} f$ we may restrict the point $x$ to the disc $D\left(0, \frac{1}{4}\right)$. Thus it suffices to prove the following.

Assume that $\lambda \in(0,1]$ and there are $M 3 \delta$-separated values $r_{j} \in\left[\frac{1}{2}, 2\right]$ and points $x_{j} \in D\left(0, \frac{1}{4}\right)$ such that $\left|E \cap C_{\delta}\left(x_{j}, r_{j}\right)\right| \geq \lambda\left|C_{\delta}\left(x_{j}, r_{j}\right)\right|$. Then

$$
M \delta \leq C\left(\frac{|E|}{\delta^{\frac{1}{6}} \lambda^{\frac{8}{3}}}\right)^{\frac{6}{5}}
$$

We can assume that $M$ is large; for $M$ smaller than any fixed constant (29) holds because $M \neq 0$ implies $|E| \gtrsim \lambda \delta$.

To prove (29) we let $\mu$ ("multiplicity") be the smallest number with the following property: there are at least $\frac{M}{2}$ values of $j$ such that

$$
\left|E \cap C_{\delta}\left(x_{j}, r_{j}\right) \cap\left\{x:\left|\left\{i: x \in C_{\delta}\left(x_{i}, r_{i}\right)\right\}\right| \leq \mu\right\}\right| \geq \frac{\lambda}{2}\left|C_{\delta}\left(x_{j}, r_{j}\right)\right|
$$

The main estimate is

$$
\mu \lesssim M^{\frac{1}{6}} \lambda^{-\frac{5}{3}}
$$

Before proving (31) we introduce some more notation, as follows. For any $t \in[\delta, 1]$ and $\epsilon \in[\delta, 1]$, let

$$
a(t, \epsilon)=C_{1}^{-1}\left(\frac{\delta}{\epsilon}\right)^{\alpha}\left(\frac{M \delta}{t}+\frac{t}{M \delta}\right)^{-\alpha}
$$

Here $\alpha$ is a sufficiently small positive constant, and $C_{1}$ is a positive constant (easily shown to exist) which is large enough that

$$
\sum_{\substack{k \geq 0 \\ l \geq 0}} a\left(2^{k} \delta, 2^{l} \delta\right)<1
$$

\footnotetext{
${ }^{4}$ The $p=2$ case was also known prior to [31]; it follows from results of Pecher [41].
} 
for all $M$ and $\delta$. Let $\bar{\lambda}(t, \epsilon)=a(t, \epsilon) \frac{\lambda}{2}, \bar{\mu}(t, \epsilon)=a(t, \epsilon) \mu, \bar{M}(t, \epsilon)=a(t, \epsilon) \frac{M}{2}$. Also, for each $i, j \in\{1, \ldots, M\}$ let

$$
\Delta_{i j}=\max \left(\delta,|| x_{i}-x_{j}|-| r_{i}-r_{j}||\right)
$$

and for each $j \in\{1, \ldots, M\}, t \in[\delta, 1], \epsilon \in[\delta, 1]$, let

$$
\begin{gathered}
S_{t, \epsilon}\left(x_{j}, r_{j}\right) \stackrel{\text { def }}{=}\left\{i: C_{\delta}\left(x_{j}, r_{j}\right) \cap C_{\delta}\left(x_{i}, r_{i}\right) \neq \emptyset, t \leq\left|r_{i}-r_{j}\right| \leq 2 t\right. \\
\left.\quad \text { and } \epsilon \leq \Delta_{i j} \leq 2 \epsilon\right\} \\
A_{t, \epsilon}\left(x_{j}, r_{j}\right) \stackrel{\text { def }}{=}\left\{x \in C_{\delta}\left(x_{j}, r_{j}\right):\left|\left\{i \in S_{t, \epsilon}\left(x_{j}, r_{j}\right): x \in C_{\delta}\left(x_{i}, r_{i}\right)\right\}\right| \geq \bar{\mu}(t, \epsilon)\right\}
\end{gathered}
$$

Lemma 3.4 There are numbers $t \in[\delta, 1]$ and $\epsilon \in[\delta, 1]$ with the following property

There are $\geq \bar{M}(t, \epsilon)$ values of $j$ such that $\left|A_{t \epsilon}\left(x_{j}, r_{j}\right)\right| \geq \bar{\lambda}(t, \epsilon)\left|C_{\delta}\left(x_{j}, r_{j}\right)\right|$

Proof This is a routine pigeonhole argument. By the minimality of $\mu$ there are at least $\frac{M}{2}$ values of $j$ such that $\left|\tilde{E}_{j}\right| \geq \frac{\lambda}{2}\left|C_{\delta}\left(x_{j}, r_{j}\right)\right|$ where

$$
\tilde{E}_{j}=E \cap C_{\delta}\left(x_{j}, r_{j}\right) \cap\left\{x:\left|\left\{i: x \in C_{\delta}\left(x_{i}, r_{i}\right)\right\}\right| \geq \mu\right\}
$$

For any such $j$ and any $x \in \tilde{E}_{j}$, (32) implies there are $t=2^{k} \delta$ and $\epsilon=2^{l} \delta$ such that $x \in A_{t \epsilon}\left(x_{j}, r_{j}\right)$. Consequently, using (32) again, for any such $j$ there are $t=2^{k} \delta$ and $\epsilon=2^{l} \delta$ such that

$$
\left|A_{t \epsilon}\left(x_{j}, r_{j}\right)\right| \geq \bar{\lambda}(t, \epsilon)\left|C_{\delta}\left(x_{j}, r_{j}\right)\right|
$$

By (32) once more, there must be a choice of $t$ and $\epsilon$ such that (34) holds for at least $\bar{M}(t, \epsilon)$ values of $j$. This finishes the proof.

We fix once and for all a pair $(t, \epsilon)$ for which the conclusion of Lemma 3.4 is valid, and will drop the $t, \epsilon$ subscripts when convenient, i.e. will denote $\bar{\lambda}(t, \epsilon)$ by $\bar{\lambda}$, etc. We split the proof of (31) into two cases:

(i) $\bar{\lambda} \geq C_{2} \sqrt{\frac{\epsilon}{t}}$

(ii) $\bar{\lambda} \leq C_{2} \sqrt{\frac{\epsilon}{t}}$

where $C_{2}$ is a sufficiently large constant.

In case (i), which is the main case, we let $S$ be the set of $M$ circles in (29), and let $\bar{S}$ be the set of at least $\bar{M}$ circles in Lemma 3.4. Let $Q$ be the set of all quadruples $\left(j, j_{1}, j_{2}, j_{3}\right)$ with $C\left(x_{j}, r_{j}\right) \in \bar{S}, C\left(x_{j_{i}}, r_{j_{i}}\right) \in S$ for $i=1,2,3$ and such that $j_{i} \in S_{t, \epsilon}\left(x_{j}, r_{j}\right)$ for each $i \in\{1,2,3\}$ and furthermore $\operatorname{dist}\left(C_{\delta}\left(x_{j}, r_{j}\right) \cap C_{\delta}\left(x_{j_{i}}, r_{j_{i}}\right), C_{\delta}\left(x_{j}, r_{j}\right) \cap C_{\delta}\left(x_{j_{k}}, r_{j_{k}}\right)\right) \geq C_{3}^{-1} \bar{\lambda}$ 
for all $i, k \in\{1,2,3\}$ with $i \neq k$. Here $C_{3}$ is a suitable constant which should be chosen before $C_{2}$.

We will make two different estimates on the cardinality of $Q$. On the one hand, the diameter bound in Lemma 3.2 implies that for fixed $j_{1}, j_{2}, j_{3}$ there are $\lesssim \frac{\epsilon}{\delta} \bar{\lambda}^{-2}$ values of $j$ such that $\left(j, j_{1}, j_{2}, j_{3}\right) \in Q$. Also it follows from the definition of $Q$ that there are $\lesssim M \min \left(M, \frac{t}{\delta}\right)^{2}$ possible choices for $\left(j_{1}, j_{2}, j_{3}\right)$ : there are at most $M$ choices for $j_{1}$, and once $j_{1}$ is fixed there are $\lesssim \min \left(M, \frac{t}{\delta}\right)$ possibilities for each of $j_{2}$ and $j_{3}$, since $\left|r_{j_{1}}-r_{j_{i}}\right| \leq\left|r_{j_{1}}-r_{j}\right|+\left|r_{j}-r_{j_{i}}\right| \leq 4 t$ for $i=2$ or 3 . We conclude that

$$
|Q| \lesssim \frac{\epsilon}{\delta} \bar{\lambda}^{-2} M \min \left(M, \frac{t}{\delta}\right)^{2}
$$

On the other hand, if we fix $j$ with $C\left(x_{j}, r_{j}\right) \in \bar{S}$ then (provided $C_{3}$ has been chosen large enough) we can find three subsets $F_{1}, F_{2}, F_{3}$ of $A_{t, \epsilon}\left(x_{j}, r_{j}\right)$ such that $\operatorname{dist}\left(F_{l}, F_{m}\right) \geq$ $2 C_{3}^{-1} \bar{\lambda}, l \neq m$, and $\left|F_{l}\right| \gtrsim \delta \bar{\lambda}$ for each $l$. For fixed $l$, we let $S_{l}$ be those indices $i \in S_{t, \epsilon}\left(x_{j}, r_{j}\right)$ such that $F_{l} \cap C_{\delta}\left(x_{i}, r_{i}\right) \neq \emptyset$. The sets $C_{\delta}\left(x_{i}, r_{i}\right), i \in S_{l}$ must cover $F_{l}$ at least $\bar{\mu}$ times. So

$$
\sum_{i \in S_{l}}\left|F_{l} \cap C_{\delta}\left(x_{i}, r_{i}\right)\right| \gtrsim \bar{\mu} \bar{\lambda} \delta
$$

For each fixed $i$ we have $\left|F_{l} \cap C_{\delta}\left(x_{i}, r_{i}\right)\right| \lesssim \frac{\delta^{2}}{\sqrt{t \epsilon}}$ by Lemma 3.1(a). Consequently

$$
\left|S_{l}\right| \gtrsim \delta^{-1} \bar{\mu} \bar{\lambda} \sqrt{t \epsilon}
$$

It is easy to see using Lemma 3.1(b) that if $i_{l} \in S_{l}$ for $l=1,2,3$ then $\left(j, i_{1}, i_{2}, i_{3}\right) \in Q$. So

$$
|Q| \gtrsim \bar{M}\left(\delta^{-1} \bar{\mu} \bar{\lambda} \sqrt{t \epsilon}\right)^{3}
$$

If we compare this with (35) we obtain

$$
\bar{\mu}^{3} \lesssim \frac{\delta^{2}}{t^{\frac{3}{2}} \epsilon^{\frac{1}{2}}} \bar{\lambda}^{-5} \min \left(M, \frac{t}{\delta}\right)^{2} \frac{M}{\bar{M}}
$$

or equivalently

$$
\mu^{3} \lesssim M^{\frac{1}{2}} \lambda^{-5} \cdot \begin{cases}a(t, \epsilon)^{-9}\left(\frac{\delta}{\epsilon}\right)^{\frac{1}{2}}\left(\frac{t}{\delta M}\right)^{\frac{1}{2}} & \text { if } M \geq \frac{t}{\delta} \\ a(t, \epsilon)^{-9}\left(\frac{\delta}{\epsilon}\right)^{\frac{1}{2}}\left(\frac{M \delta}{t}\right)^{\frac{3}{2}} & \text { if } M \leq \frac{t}{\delta}\end{cases}
$$

The expression in the brace is bounded by a constant by the definition of $a(t, \epsilon)$, provided $\alpha<\frac{1}{18}$. So we have proved (31) in case (i). 
In case (ii), we fix $j$ with $C\left(x_{j}, r_{j}\right) \in \bar{S}$ and make the trivial estimate $\left|S_{t \epsilon}\left(x_{j}, r_{j}\right)\right| \lesssim$ $\min \left(M, \frac{t}{\delta}\right)$. It follows that

$$
\begin{aligned}
\bar{\mu} \bar{\lambda} \delta & \lesssim \sum_{i \in S_{t} \epsilon\left(x_{j}, r_{j}\right)}\left|C_{\delta}\left(x_{j}, r_{j}\right) \cap C_{\delta}\left(x_{i}, r_{i}\right)\right| \\
& \lesssim \min \left(M, \frac{t}{\delta}\right) \frac{\delta^{2}}{\sqrt{t \epsilon}}
\end{aligned}
$$

where we used Lemma 3.1(a). Thus $\bar{\mu} \lesssim \bar{\lambda}^{-1} \sqrt{\frac{t}{\epsilon}} \min \left(\frac{M \delta}{t}, 1\right)$. Using the hypothesis (ii) we therefore have

$$
\bar{\mu} \lesssim \bar{\lambda}^{-\frac{5}{3}}\left(\frac{t}{\epsilon}\right)^{\frac{1}{6}} \min \left(\frac{M \delta}{t}, 1\right)
$$

i.e.

$$
\mu \lesssim \lambda^{-\frac{5}{3}} M^{\frac{1}{6}} \cdot \begin{cases}a(t, \epsilon)^{-\frac{8}{3}}\left(\frac{\delta}{\epsilon}\right)^{\frac{1}{6}}\left(\frac{t}{\delta M}\right)^{\frac{1}{6}} & \text { if } M \geq \frac{t}{\delta} \\ a(t, \epsilon)^{-\frac{8}{3}}\left(\frac{\delta}{\epsilon}\right)^{\frac{1}{6}}\left(\frac{M \delta}{t}\right)^{\frac{5}{6}} & \text { if } M \leq \frac{t}{\delta}\end{cases}
$$

The expression in the brace is bounded by a constant provided $\alpha<\frac{1}{16}$, so we have proved (31).

Completion of proof of Proposition 3.3 Let $\tilde{E}=\left\{i: x \in C_{\delta}\left(x_{i}, r_{i}\right) \mid \leq \mu\right\}$ With notation as above we have

$$
\begin{aligned}
|E| \geq|\tilde{E}| & \geq \mu^{-1} \sum_{j}\left|\tilde{E} \cap C_{\delta}\left(x_{j}, r_{j}\right)\right| \\
& \gtrsim \mu^{-1} M \lambda \delta \\
& \gtrsim \lambda^{\frac{8}{3}} M^{\frac{5}{6}} \delta
\end{aligned}
$$

by (31). Consequently $(M \delta)^{\frac{5}{6}} \lesssim \frac{|E|}{\delta^{\frac{1}{6}} \lambda^{\frac{8}{3}}}$ and the proposition is proved.

Further remarks 3.2. We mention some other recent related work. Schlag [43] found an essentially optimal $L^{p} \rightarrow L^{q}$ estimate in the context of Bourgain's theorem. If we define

$$
\mathcal{M}_{\delta} f(x)=\sup _{1 \leq r \leq 2} \frac{1}{\left|C_{\delta}(x, r)\right|} \int_{C_{\delta}^{(x, r)}}|f|
$$

then there is an estimate

$$
\forall \epsilon \exists C_{\epsilon}\left\|\mathcal{M}_{\delta} f\right\|_{5} \lesssim C_{\epsilon} \delta^{-\epsilon}\|f\|_{\frac{5}{2}}
$$

and modulo $\delta^{-\epsilon}$ factors all possible $L^{p} \rightarrow L^{q}$ bounds for $\mathcal{M}_{\delta}$ follow by interpolation from this one. Alternate proofs and further related results are in [46], [61] and [44]. On the other hand a number of endpoint questions remain open. The best known is the restricted weak type $(2,2)$ version of Bourgain's theorem. 
3.3. A more central open question is the so-called local smoothing conjecture [48], [36] in $2+1$ dimensions. See section 4 below. This is a problem "with cancellation" and likely not susceptible to purely combinatorial methods without additional input. On the other hand, it would imply (18) with $p=4$ via the Sobolev embedding theorem and is therefore close to including some of the results of [17]. This means perhaps that a proof not involving any combinatorics would have to contain a significant new idea.

3.4. One can give a discrete heuristic for the Kakeya problem analogous to the one for the BRK sets problem. What follows is an observation of Schlag and the author.

There is a substantial literature on incidence problems for lines in $\mathbb{R}^{3}$; these problems appear to be quite difficult and are largely open. One relevant paper is Sharir [47], where the following problem is considered:

Let $\left\{\ell_{j}\right\}_{j=1}^{N}$ be lines in $\mathbb{R}^{3}$ and define a joint to be a point where three noncoplanar $\ell_{j}$ 's intersect. Then how many joints can there be?

If $\mathcal{J}$ is the set of joints then as is discussed in [47] the natural conjecture is $|\mathcal{J}| \lesssim N^{\frac{3}{2}}$, which would be sharp by taking $\approx \sqrt{N}$ planes parallel to each of three given planes and considering the lines formed by intersecting two of the planes; any point where three planes intersect will be a joint. The "easy" bound in this problem is $|\mathcal{J}| \lesssim N^{\frac{7}{4}}$ which is proved in [14] using a suitable version of (3). The bound $\forall \epsilon \exists C_{\epsilon}:|\mathcal{J}| \leq C_{\epsilon} N^{\frac{23}{14}+\epsilon}$ is proved in [47] using similar techniques to [17].

The heuristic is that a bound $|\mathcal{J}| \lesssim N^{\alpha}$ should imply that (in $\mathbb{R}^{3}$ ) $\operatorname{dim}$ (Kakeya) $\geq \frac{\alpha}{\alpha-1}$. Namely, define a $\mu$-fold point in an arrangement of $N$ lines to be a point where at least $\mu$ lines intersect with (say) no more than half of these lines belonging to any given 2-plane. Then any bound of the form $|\mathcal{J}| \lesssim N^{\alpha}$ leads to a corresponding bound $\left|\mathcal{P}_{\mu}\right| \lesssim\left(\frac{N \log \mu}{\mu}\right)^{\alpha}$ where $\mathcal{P}_{\mu}$ is the set of $\mu$-fold points. This may be seen (rigorously) as follows: let $\mathcal{P}_{\mu}$ be the set of $\mu$-fold points in the arrangement. Let $A$ be a large constant and take a random sample of the $N$ lines according to the following rule: each line belongs to the sample independently and with probability $\frac{A \log \mu}{\mu}$. Then with high probability the sample has cardinality $\lesssim \frac{N \log \mu}{\mu}$. Furthermore, it is not hard to show that any point of $\mathcal{P}_{\mu}$ will be a joint for the lines in the sample with probability at least $1-\mu^{-B}$, where $B$ is large if $A$ is large. It follows that with high probability at least half the points of $\mathcal{P}_{\mu}$ will be joints for the sample, hence $\left|\mathcal{P}_{\mu}\right| \lesssim\left(\frac{N \log \mu}{\mu}\right)^{\alpha}$.

Now the heuristic part of the argument: suppose we have a Kakeya set $E$ with (say, Minkowski) dimension $\beta$. Fix $\delta$ and take a $\delta$-separated set of directions and a line segment in each direction contained in $E$; this gives an arrangement of $\approx \delta^{-2}$ lines $\left\{\ell_{j}\right\}$. Let $E^{\delta}$ be the $\delta$-neighborhood of $E$; thus $\left|E^{\delta}\right| \approx \delta^{\beta}$, so $E^{\delta}$ is made up of roughly $\delta^{-\beta} \delta$-discs. A typical point in the $\delta$-neighborhood of $E$ should belong to roughly $\delta^{-(3-\beta)} \delta$-neighborhoods of $\ell_{j}$ 's, since otherwise the "low multiplicity" arguments discussed e.g. in section 2 would show easily that $\left|E^{\delta}\right|>>\delta^{\beta}$. Hence if we ignore the distinction between points and 
$\delta$-discs then we are dealing with an arrangement of $\delta^{-2}$ lines with $\delta^{-\beta} \delta^{-(3-\beta)}$-fold points. We conclude that up to logarithmic factors

$$
\delta^{-\beta} \lesssim\left(\frac{\delta^{-2}}{\delta^{-(3-\beta)}}\right)^{\alpha}
$$

i.e. $\beta \geq \frac{\alpha}{\alpha-1}$.

Under this heuristic the result of [47] would correspond to an improvement over $\frac{5}{2}$ on Kakeya, and the fact that the joints problem is open would seem to indicate that questions 1 and 2 are quite difficult even on a combinatorial level, if in fact the answers are affirmative. In this connection, we note that Schlag [45] has proved an analogue of the 3-circle lemma in this context and has used it to give an alternate proof of the result $\operatorname{dim}($ Kakeya $) \geq \frac{7}{3}$ (originally due to Bourgain [7]) which corresponds to the result from [14] via $\frac{7}{3}=\frac{7 / 4}{7 / 4-1}$. However, it is not easy to put the argument of [47] into the continuum and the author believes that in contrast to the situation considered in [61] it may not be possible to do this in a reasonably straightforward way.

A further remark is that special cases of the three dimensional Kakeya problem correspond to results analogous to [61] with circles replaced by families of curves satisfying the cinematic curvature condition from [48]. For example, the case of sets invariant by rotations around an axis is a problem of this type as is discussed in [31].

\section{Oscillatory integrals and Kakeya}

It seemed appropriate to include a discussion of the basic open problems in harmonic analysis connected with Kakeya, but we will not attempt a complete survey and will not say anything about the proofs of the deeper results. We will just state some well-known open problems and show how they lead to questions 1 and 2 .

Let $\hat{f}$ be the Fourier transform and if $m$ is a given function, then let $T_{m} f$ be the corresponding multiplier operator,

$$
\widehat{T_{m} f}=m \hat{f}
$$

Two longstanding problems in $L^{p}$ harmonic analysis are the following:

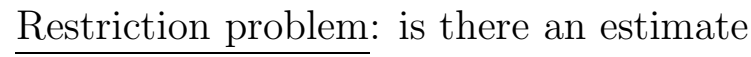

$$
\|\widehat{f d \sigma}\|_{p} \lesssim\|f\|_{L^{p}(d \sigma)}
$$

for all $p>\frac{2 n}{n-1}$, where $\sigma$ is surface measure on the unit sphere $S^{n-1} \subset \mathbb{R}^{n}$ ?

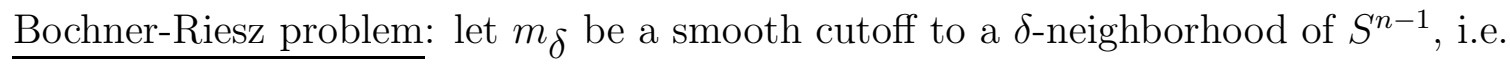

$$
m_{\delta}(\xi)=\phi\left(\delta^{-1}(1-|\xi|)\right)
$$


where $\phi \in C_{0}^{\infty}(\mathbb{R})$ is supported in $\left(-\frac{1}{2}, \frac{1}{2}\right)$. Then is there an estimate

$$
\forall \epsilon \exists C_{\epsilon}:\left\|T_{m} f\right\|_{p} \leq \delta^{-\epsilon}\|f\|_{p}
$$

when $p \in\left[\frac{2 n}{n+1}, \frac{2 n}{n-1}\right]$ ?

Both these problems can be formulated in a number of different ways; the formulations we have given are not the original ones but are well-known to be equivalent to them. In fact it would also be equivalent to prove (37) in the weaker form $\|\widehat{f d \sigma}\|_{p} \lesssim\|f\|_{\infty}, p>\frac{2 n}{n-1}$. This is a consequence of the Stein-Nikisin theory as is pointed out in [7], section 6 .

A third problem of more recent vintage [48] is

Local smoothing Let $u$ be the solution of the initial value problem for the wave equation in $n$ space dimensions,

$$
\square u=0, u(\cdot, 0)=f, \frac{\partial u}{\partial t}(\cdot, 0)=0
$$

Then is there an estimate

$$
\forall \epsilon>0 \exists C_{\epsilon}:\|u\|_{L^{p}\left(\mathbb{R}^{n} \times[1,2]\right)} \leq C_{\epsilon}\|f\|_{p, \epsilon}
$$

when $p \in\left[2, \frac{2 n}{n-1}\right]$ ? Here $\|\cdot\|_{p, \epsilon}$ is the inhomogeneous $L^{p}$ Sobolev norm with $\epsilon$ derivatives.

In all these problems it is well-known that the exponent $\frac{2 n}{n-1}$ would be optimal. See [52]. For example, in the last problem this may be seen by considering focussing solutions where $f$ is spread over a $\delta$-neighborhood of the unit sphere and $u(\cdot, t)$ is mostly concentrated on a $\delta$-disc when $t \in(1,1+\delta)$.

When $n=2$, estimate (37) was proved by Fefferman and Stein and then (38) by Carleson and Sjolin, in the early 1970's (see [52]). Estimate (39) is open even when $n=2$ however; the known partial results on $L^{4}\left(\mathbb{R}^{2}\right)$ correspond to loss of $\frac{1}{8}$ derivatives ([36]; an improvement to loss of $\frac{1}{8}-\epsilon$ derivatives appears implicit in [12], p. 60). In general dimensions, the following implications are known.

$$
(39) \Rightarrow(38) \Rightarrow(37) \Rightarrow(2)
$$

The first implication is due to Sogge, the second which is deeper is due to Tao [58], and Carbery [13] had shown earlier that the second implication can be reversed in a slightly different context (replace spheres by paraboloids). We refer to [58] for further discussion. Here though we will only be concerned with the last implication which makes the connection with the Kakeya problem. Essentially this is due to Fefferman [23], another relevant reference is [3] and the result as presented here is from [10]. A basic open problem in the area is to what extent the last implication can be reversed. An alternate proof of 
the two dimensional Carleson-Sjolin result along these lines was given by Cordoba [18]. In three or more dimensions, progress on this problem was initiated by Bourgain (see [10]) who obtained a numerology between partial results which however does not show that (2) would imply (37). For a recent improvement in the numerology see [38] and [59].

A problem of a somewhat different nature is

Montgomery's conjecture Assume $T \leq N^{2}$. Consider a Dirichlet series,

$$
D(s)=\sum_{n=1}^{N} a_{n} n^{i s}
$$

where $\left\|\left\{a_{n}\right\}\right\|_{\ell^{\infty}} \leq 1$. Let $\mathcal{T}$ be a 1 -separated subset of $[0, T]$. Then

$$
\forall \epsilon \exists C_{\epsilon}: \sum_{t \in \mathcal{T}}|D(t)|^{2} \leq N^{\epsilon}(N+|\mathcal{T}|) N
$$

An easy consequence (or reformulation) would be that

$$
\forall \epsilon \exists C_{\epsilon}: \int_{E}|D(t)|^{2} d t \leq N^{\epsilon}(N+|E|) N
$$

if $E \subset[0, T]$ with the stated hypotheses on $T$ and $D(s)$. This is an estimate on the measure of the set of large values of $D(s)$ and would also imply estimates of $L^{p}$ norms with $p>2$. See [9] and e.g. [37] for these remarks as well as some discussion of the relationship between (40) and open problems in analytic number theory. Estimate (40) can perhaps be thought of as an analogue of (37) where the oscillatory sum operator $\left\{a_{n}\right\} \rightarrow D(s)$ replaces the extension operator $f \rightarrow \widehat{f d \sigma}$. Bourgain [9] showed that (40) is again related to the Kakeya problem.

In the rest of this article, we will discuss implications of this type, i.e.

$$
\text { oscillatory integral estimates } \Rightarrow \text { Kakeya estimates }
$$

We first show that (37) implies (2), and will record the corresponding implications between partial results. Let us recall the results that would follow from (37) using Holder's inequality and interpolation with the trivial bound $\|\widehat{f d \sigma}\|_{\infty} \leq\|f\|_{L^{1}(d \sigma)}$, say

$$
\|\widehat{f d \sigma}\|_{q} \lesssim\|f\|_{L^{p}(d \sigma)}, p<\frac{2 n}{n-1}, q>\frac{n+1}{n-1} p^{\prime}
$$

This bound for $p \leq 2$ (plus its endpoint version where $q=\frac{n+1}{n-1} p^{\prime}$ ) is a well-known theorem proved by Stein and Tomas in the 1970's and the case $p=q<2 \frac{n+1}{n-1}+\epsilon$ for suitable $\epsilon>0$ 
was proved more recently by Bourgain [7] using considerations related to question 2. See [52] and [10].

Proposition 4.1 Assume (41) holds for a given $p \geq 2$ and $q \geq 2$. Then, with $r=\left(\frac{q}{2}\right)^{\prime}$ and $s=\left(\frac{p}{2}\right)^{\prime}$, the restricted weak type $(r, s)$ norm of the Kakeya maximal operator is $\lesssim \delta^{-2\left(\frac{n}{r}-1\right)}$. Consequently the Hausdorff dimension of Kakeya sets is $\geq 2 r-n=\frac{2 q}{q-2}-n$. In particular (37) implies (2).

Proof First let $\left\{T_{j}\right\}_{j=1}^{N}, T_{j}=T_{e_{j}}^{\delta}\left(a_{j}\right)$ be any collection of $\delta$-tubes with $\delta$-separated directions $e_{j}$. Let $\tilde{T}_{j}=\left\{x \in \mathbb{R}^{n}: \delta^{2} x \in T_{j}\right\}$ be the dilation of $T_{j}$ by a factor $\delta^{-2}$, and let $\chi_{j}$ and $\tilde{\chi}_{j}$ be the characteristic functions of $T_{j}$ and $\tilde{T}_{j}$ respectively. Let $C_{j}$ be a spherical cap with radius $\approx \delta$ centered at $e_{j}$, e.g. $C_{j}=\left\{e \in S^{n-1}: e \cdot e_{j} \geq 1-C^{-1} \delta^{2}\right\}$ where $C$ is a suitable constant. Take a bump function supported in $C_{j}$, say $\phi_{j} \in C_{0}^{\infty}\left(C_{j}\right)$ with $\left\|\phi_{j}\right\|_{\infty}=1, \phi_{j} \geq 0$ and $\left\|\phi_{j}\right\|_{1} \approx \delta^{n-1}$, and let $\psi_{j}(\xi)=e^{2 \pi i \xi \cdot \delta^{-2} a_{j}} \phi_{j}(\xi)$. If $x \in \tilde{T}_{j}$, then the integral

$$
\widehat{\psi_{j} d \sigma}(x)=\int_{S^{n-1}} \psi_{j}(\xi) e^{-2 \pi i \xi \cdot x} d \xi=e^{-2 \pi i e_{j} \cdot\left(x-\delta^{-2} a_{j}\right)} \int_{C_{j}} \phi_{j}(\xi) e^{-2 \pi i\left(\xi-e_{j}\right) \cdot\left(x-\delta^{-2} a_{j}\right)} d \xi
$$

defining $\widehat{\psi_{j} d \sigma}(x)$ involves no cancellation, so

$$
\left|\widehat{\psi_{j} d \sigma}\right| \gtrsim \delta^{n-1} \tilde{\chi}_{j}
$$

Now consider the function $f=\sum_{j} \epsilon_{j} \psi_{j}$ where the $\epsilon_{j}$ are random \pm 1 's. Since the supports of the $\psi_{j}$ are disjoint we have

$$
\|f\|_{L^{p}\left(S^{n-1}\right)} \lesssim\left(N \delta^{n-1}\right)^{\frac{1}{p}}
$$

and therefore, by the assumption (41),

$$
\|\widehat{f d \sigma}\|_{q} \lesssim\left(N \delta^{n-1}\right)^{\frac{1}{p}}
$$

for any choice of \pm . On the other hand, if we let $\mathbb{E}$ denote expectation with respect to the choices of \pm , then by Khinchin's inequality and (42)

$$
\mathbb{E}\left(|\widehat{f d \sigma}|^{q}\right) \gtrsim \delta^{q(n-1)}\left(\sum_{j} \tilde{\chi}_{j}\right)^{\frac{q}{2}}
$$

pointwise. If we integrate this inequality and compare with (43) we obtain

$$
\delta^{q(n-1)}\left\|\sum_{j} \tilde{\chi}_{j}\right\|_{\frac{q}{2}}^{\frac{q}{2}} \lesssim\left(N \delta^{n-1}\right)^{\frac{q}{p}}
$$


Rescaling by $\delta^{2}$, then taking $\frac{q}{2}$ th roots,

$$
\delta^{2(n-1)-\frac{4 n}{q}}\left\|\sum_{j} \chi_{j}\right\|_{\frac{q}{2}} \lesssim\left(N \delta^{n-1}\right)^{\frac{2}{p}}
$$

Now let $E$ be a set, $f=\chi_{E}$ and

$$
\Omega=\left\{e: f_{\delta}^{*}(e) \geq \lambda\right\}
$$

Let $\left\{e_{j}\right\}_{j=1}^{N}$ be a maximal $\delta$-separated subset of $\Omega$ and for each $j$ choose a $\delta$-tube $T_{j}$ as above with $\left|E \cap T_{j}\right| \geq \lambda\left|T_{j}\right|$. Then

$$
\begin{aligned}
N \lambda \delta^{n-1} & \leq \sum_{j}\left|T_{j} \cap E\right| \\
& \lesssim|E|^{1-\frac{2}{q}}\left\|\sum_{j} \chi_{j}\right\|_{\frac{q}{2}} \\
& \lesssim|E|^{1-\frac{2}{q}}\left(N \delta^{n-1}\right)^{\frac{2}{p}} \delta^{-2(n-1)+\frac{4 n}{q}}
\end{aligned}
$$

Using (6) this implies that

$$
|\Omega|^{1-\frac{2}{p}} \lesssim \lambda^{-1}|E|^{1-\frac{2}{q}} \delta^{-2(n-1)+\frac{4 n}{q}}
$$

i.e. $|\Omega|^{\frac{1}{s}} \lesssim \lambda^{-1}|E|^{\frac{1}{r}} \delta^{-2\left(\frac{n}{r}-1\right)}$ which is the bound that was claimed. The dimension statement in the proposition then follows from Lemma 1.6, and the last statement also follows by letting $p \rightarrow \frac{2 n}{n-1}$ and using well-known formal arguments.

$\underline{\text { Remarks }}$ 4.1. The original Fefferman construction was of course a counterexample; essentially he showed

If the disc multiplier were bounded on $L^{p}$ with $p \neq 2$, then families of tubes with the property in Remark 1.2 could not exist.

The paper [3] applies the argument from [23] to the restriction problem in the above way but the result is again formulated as a counterexample. The formulation as an implication concerning the maximal function is from [10].

4.2. We present another application of the Fefferman construction which shows the following.

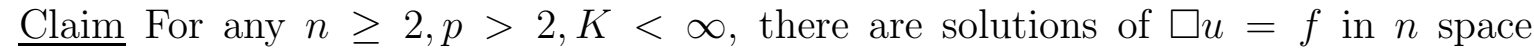
dimensions, with $\|f\|_{\infty} \leq 1, \operatorname{supp} f \subset D(0,100) \times[0,1]$, and

$$
\int_{2}^{3}\left\|\frac{\partial u}{\partial t}(\cdot, t)\right\|_{L^{p}\left(\mathbb{R}^{n}\right)} d t>K
$$


The analogous statement with the $x$-gradient replacing the $t$-derivative can be proved in a similar way. The statement can be understood as follows: the energy estimate for the wave equation implies via Duhamel's principle that $\|\nabla u(\cdot, t)\|_{2} \lesssim\|f\|_{2}$ if say $t \in(2,3)$ and $f$ is supported in $\mathbb{R}^{n} \times[0,1]$. The claim says that there can be no such estimate in $L^{p}, p>2$, even if one is willing to average in $t$ as in (39) and to restrict to bounded $f$ with compact support. The claim was proved by the author after discussions with S. Klainerman but it is very close to the surface given [23]. The analogous statement for the initial value problem is essentially that (39) fails if the $W^{p \epsilon}$ norm is replaced by the $L^{p}$ norm on the right hand side; this is a formal consequence of [23] as was probably first observed by Sogge.

The construction below by no means rules out an estimate with loss of $\epsilon$ derivatives. In fact the estimate $\int_{2}^{3}\left\|\frac{\partial u}{\partial t}(\cdot, t)\right\|_{L^{p}\left(\mathbb{R}^{n}\right)}^{p} d t \lesssim\|f\|_{p, \epsilon}^{p}$ with $2<p \leq \frac{2 n}{n-1}$ and any $\epsilon>0$ would follow from (39) via Duhamel.

Proof of the claim If $x \in \mathbb{R}^{n}$ then we will use the notation $x=\left(x_{1}, \bar{x}\right), \bar{x} \in \mathbb{R}^{n-1}$.

For an appropriate constant $C$ and any small enough $\delta$ there is a solution of $\square u=f$ with

$$
\|f\|_{\infty} \leq 1, \operatorname{supp} f \subset\left\{(x, t): 0 \leq t \leq 1,0 \leq x_{1} \leq 1,|\bar{x}| \leq \delta\right\}
$$

and

$$
\left|\frac{\partial u}{\partial t}\right| \geq C^{-1} \text { when } 2 \leq t \leq 3, x \in Y^{t}
$$

where $Y^{t}$ is a subset of $\left\{x \in \mathbb{R}^{n}: 2 \leq x_{1} \leq 3,|\bar{x}| \leq \delta\right\}$ with measure $\geq C^{-1} \delta^{n-1}$.

This is essentially just the fact that there are high frequency solutions of the wave equation travelling in a single direction tangent to the light cone, which implies we can find $f$ with the indicated support and such that $u$ restricted to $2 \leq t \leq 3$ is also mostly concentrated where $|\bar{x}| \lesssim \delta$. The conclusion then corresponds to conservation of energy.

A rigorous argument can be based on the explicit choice

$$
f(x, t)=e^{2 \pi i N\left(x_{1}-t\right)} \phi\left(x_{1}\right) \psi\left(\delta^{-1} \bar{x}\right) \chi(t)
$$

where $N$ is very large, $\phi, \psi, \chi$ are fixed nonnegative $C_{0}^{\infty}$ functions, $\psi(0)=1, \operatorname{supp} \psi \subset$ $D(0,1), \operatorname{supp} \phi=\operatorname{supp} \chi=[0,1]$ and $\phi$ and $\chi$ are strictly positive on $(0,1)$. Let $u$ be the corresponding solution of the wave equation. Then $u$ is given by the formula

$$
u(x, t)=\int e^{2 \pi i x \cdot \xi} \frac{\sin (2 \pi(t-s)|\xi|)}{2 \pi|\xi|} e^{-2 \pi i N s} \hat{\phi}\left(\xi_{1}-N\right) \delta^{n-1} \hat{\psi}(\delta \bar{\xi}) \chi(s) d \xi d s
$$

One can differentiate for $\mathrm{t}$ and then evaluate the resulting integral precisely enough to obtain (45) in the region $\left|x_{1}-t\right| \leq \frac{1}{2},|\bar{x}| \leq C^{-1} \delta$. We omit the details. 
If $E$ is a set in space-time then we will use the notation $E^{t}=\left\{x \in \mathbb{R}^{n}:(x, t) \in E\right\}$. By Remarks 1.2 and 1.3 we can find disjoint $\delta$-tubes $\left\{T_{j}\right\}_{j=1}^{M}$ in $\mathbb{R}^{n}\left(M \approx \delta^{-(n-1)}\right)$ such that the tubes $\tilde{T}_{j}$ obtained by translating the $T_{j}$ 's by 2 units along their axes are all contained in a set with small measure $a(\delta)$. Let $\Pi_{j}=T_{j} \times[0,1] \subset \mathbb{R}^{n} \times \mathbb{R}$, and let $\tilde{\Pi}_{j}=\tilde{T}_{j} \times[2,3]$. By the first step of the proof there are functions $u_{j}$ and $f_{j}, \square u_{j}=f_{j}$, with $f_{j}$ supported on $\Pi_{j},\left\|f_{j}\right\|_{\infty} \leq 1$, and $\left|\frac{\partial u_{j}}{\partial t}\right| \geq$ const on a subset $Y_{j} \subset \tilde{\Pi}_{j}$ which satisfies $\left|Y_{j}^{t}\right| \approx \delta^{n-1}$ for each $t \in(2,3)$. Let $Z=\cup_{j} Y_{j}$; then $\left|Z^{t}\right| \lesssim a(\delta)$ for any $t \in(2,3)$.

Let $\left\{\epsilon_{j}\right\}$ be random \pm 1 's. Consider the functions $u=\sum_{j} \epsilon_{j} u_{j}, f=\sum_{j} \epsilon_{j} f_{j}$, which satisfy $\square u=f$. The $\Pi_{j}$ 's are disjoint, so $\|f\|_{\infty} \leq 1$ for any choice of $\epsilon_{j}$ 's. On the other hand, by Holder's and Khinchin's inequalities, for any fixed $t \in(2,3)$ we have

$$
\begin{aligned}
\left.\mathbb{E}\left(\int_{Z^{t}}\left|\frac{\partial u}{\partial t}(x, t)\right|^{p} d x\right)^{\frac{2}{p}}\right) & \left.\gtrsim a(\delta)^{-\left(1-\frac{2}{p}\right)} \mathbb{E}\left(\int_{Z^{t}}\left|\frac{\partial u}{\partial t}(x, t)\right|^{2} d x\right)\right) \\
& =a(\delta)^{-\left(1-\frac{2}{p}\right)} \int_{Z^{t}} \sum_{j}\left|\frac{\partial u_{j}}{\partial t}(x, t)\right|^{2} d x \\
& \gtrsim a(\delta)^{-\left(1-\frac{2}{p}\right)} \sum_{j}\left|Y_{j}^{t}\right| \approx a(\delta)^{-\left(1-\frac{2}{p}\right)}
\end{aligned}
$$

which shows there can be no estimate of the form

$$
\left(\int_{2}^{3}\left\|\frac{\partial u}{\partial t}(\cdot, t)\right\|_{L^{p}\left(\mathbb{R}^{n}\right)}^{2} d t\right)^{\frac{1}{2}} \leq C\|f\|_{\infty}
$$

with $p>2$ when $f$ has support in $D(0,100) \times[0,1]$. We then also obtain $(44)$, since an estimate to $L_{t}^{1}\left(L_{x}^{p}\right)$ would imply an estimate to $L_{t}^{2}\left(L_{x}^{q}\right)\left(\frac{1}{q}=\frac{1}{2}\left(\frac{1}{2}+\frac{1}{p}\right)\right)$ by interpolation with the energy estimate to $L_{t}^{\infty}\left(L_{x}^{2}\right)$.

We now discuss the argument from [9] relating (40) to (2). Bourgain showed there that Montgomery's conjecture if true would imply Kakeya sets have full dimension and a bound like (2) with a different $L^{p}$ exponent. We reworked the argument a bit for expository reasons and in order to obtain the precise result $(40) \Rightarrow(2)$.

The logic is that (40) implies a Kakeya type statement for arithmetic progressions, which in turn implies (2) for all $n$. Thus the implication $(40) \Rightarrow(2)$ follows by combining Propositions 4.2 and 4.3 below.

If $\nu \in(0,1), \beta \in \mathbb{R}$, then we denote

$$
P_{\nu}^{\delta}(\beta)=\{x \in[0,1]:|x-(j \nu+\beta)|<\delta \text { for some } j \in \mathbb{Z}\}
$$

i.e. $P_{\nu}^{\delta}(\beta)$ is the $\delta$-neighborhood of the arithmetic progression with modulus $\nu$ which contains $\beta$, intersected with $[0,1]$. 
Proposition 4.2 Assume the conjecture (40). Then for any $\epsilon$ there is $C_{\epsilon}$ such that the following holds.

(*) Fix $\eta \in(0,1), \delta \in(0, \eta)$. Let $E \subset[0,1]$ be such that

$$
\forall \nu \in Y \exists \beta \in \mathbb{R}:\left|P_{\nu}^{\delta}(\beta) \cap E\right| \geq \lambda\left|P_{\nu}^{\delta}(\beta)\right|
$$

where $\lambda \in(0,1]$ satisfies $\lambda \geq C_{\epsilon}\left(\frac{\delta^{2}}{\eta}\right)^{-\epsilon} \cdot \eta$, and where $Y$ is a subset of $\left(\frac{\eta}{2}, \eta\right)$ with $|Y| \geq \frac{\eta}{100}$. Then

$$
|E| \geq C_{\epsilon}^{-1}\left(\frac{\delta^{2}}{\eta}\right)^{\epsilon} \lambda
$$

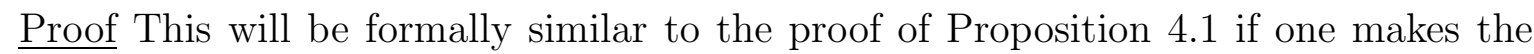
analogy

line segment $\longleftrightarrow$ arithmetic progression

spherical cap $\longleftrightarrow$ interval of integers

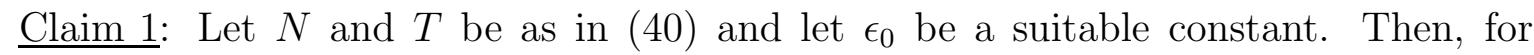
$\nu \in\left[\frac{N}{2}, N\right]$ and $\beta \in \mathbb{R}$, the Dirichlet series

$$
d(s)=\sum_{n:|n-[\nu]| \leq \epsilon_{0} \frac{N}{\sqrt{T}}} e^{-i \frac{\beta}{[\nu]}(n-[\nu])} n^{i s}
$$

satisfies

$$
|d(s)| \gtrsim \frac{N}{\sqrt{T}}
$$

when $s \leq T$ and $\operatorname{dist}(s, 2 \pi \nu \mathbb{Z}+\beta) \leq \sqrt{T}$.

Proof This is the "short sum" construction in [9]. Assume at first that $\nu \in \mathbb{Z}$. The Taylor expansion of the logarithm function shows that $\left.n^{i s}=\nu^{i s} e^{i s\left(\frac{n-\nu}{\nu}+\mathcal{O}\left(\frac{n-\nu}{\nu}\right)^{2}\right)}\right)$, so that $e^{-i \frac{\beta}{\nu}(n-\nu)} n^{i s}=\nu^{i s} e^{i(n-\nu)) \frac{s-\beta}{\nu}+i s \mathcal{O}\left(\left(\frac{n-\nu}{\nu}\right)^{2}\right)}$. Thus the sum (47) involves no cancellation and the bound follows immediately. The general case (i.e. $\nu \notin \mathbb{Z}$ ) follows by replacing $\nu$ by $[\nu]$ and noting that this does not significantly affect the hypothesis on $s$, since if $\operatorname{dist}(s, 2 \pi \nu \mathbb{Z}+\beta) \leq \sqrt{T}$ then $\operatorname{dist}(s, 2 \pi[\nu] \mathbb{Z}+\beta) \leq \sqrt{T}+C \frac{T}{N} \lesssim \sqrt{T}$.

We therefore define $\tilde{P}_{\nu}(\beta)=\{x \in[0, T]: \operatorname{dist}(s, 2 \pi \nu \mathbb{Z}+\beta) \leq \sqrt{T}\}$. We also fix a number $\epsilon>0$ and let $C_{\epsilon}$ be a suitable constant. 
Claim 2 Assume (40) and let $E$ be a subset of $[0, T]$ with the following property: there is a set $Y \subset\left[\frac{N}{2}, N\right]$ with $|Y| \geq \frac{N}{100}$, such that for any $\nu \in Y$ there is $\beta=\beta(\nu) \in \mathbb{R}$ such that $\left|E \cap \tilde{P}_{\nu}(\beta)\right| \geq \lambda\left|\tilde{P}_{\nu}(\beta)\right|$. Then

$$
|E| \geq C_{\epsilon}^{-1} N^{-\epsilon} T \lambda
$$

provided $\lambda \geq C_{\epsilon} N^{\epsilon} \frac{N}{T}$.

Proof Let $\epsilon_{0}$ be as in claim 1 , choose a maximal $2 \epsilon_{0} \frac{N}{\sqrt{T}}+1$-separated subset $\left\{\nu_{j}\right\}_{j=1}^{M} \subset$ $Y$, denote $\tilde{P}_{j}=\tilde{P}_{\nu_{j}}\left(\beta_{j}\right)$ and let $\chi_{j}$ be the characteristic function of $\tilde{P}_{j}$. Construct Dirichlet

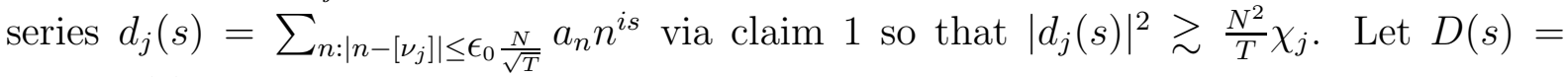
$\sum_{j} \epsilon_{j} d_{j}(s)$ where the $\epsilon_{j}$ are random \pm 1 's. By Khinchin's inequality

$$
\mathbb{E}\left(|D(s)|^{2}\right) \gtrsim \frac{N^{2}}{T} \sum_{j=1}^{M} \chi_{j}
$$

pointwise. On the other hand the coefficient intervals for the $d_{j}$ are disjoint so for any choice of $\pm 1, D(s)$ will be a Dirichlet series with coefficients bounded by 1 . Integrating (49) over $E$ and using (40), we obtain

$$
\begin{aligned}
\frac{N^{2}}{T} \sum_{j=1}^{M}\left|E \cap \tilde{P}_{j}\right| & \lesssim \mathbb{E}\left(\int_{E}|D(s)|^{2}\right) \\
& \lesssim N^{\epsilon}(N+|E|) N
\end{aligned}
$$

We have $M \approx \sqrt{T}$, and for each $j$ we have $\left|E \cap \tilde{P}_{j}\right| \geq \lambda \frac{T^{\frac{3}{2}}}{N}$. So we obtain $T \lambda \lesssim N^{\epsilon}(N+|E|)$. Under the stated hypothesis on $\lambda$ this implies (48).

Proposition 4.2 follows from claim 2 by rescaling: set $T=\delta^{-2}$ and $N=\eta \delta^{-2}$, and make the change of variables $x \rightarrow T x, \nu \rightarrow T \nu$.

Proposition 4.3 If $(*)$ holds then (2) holds in all dimensions $n$.

Proof We first observe that $(*)$ implies a generalization of itself via a well-known formal argument (one of the arguments in the Stein-Nikisin theory, see [50], p. 146). Namely, drop the hypothesis $|Y| \geq \frac{\eta}{100}$. Then, with the other hypotheses unchanged,

$$
|E| \gtrsim \lambda \frac{|Y|}{\eta}\left(\frac{\delta^{2}}{\eta}\right)^{\epsilon}
$$

To prove (50), let $\rho Y$ be the dilation of $Y$ by $\rho$. One can find numbers $\left\{\rho_{j}\right\}_{j=1}^{M} \subset\left(\frac{1}{2}, 2\right)$, where $M \approx \frac{\eta}{|Y|}$, so that $\tilde{Y} \stackrel{\text { def }}{=} \cup_{j} \rho_{j} Y$ satisfies $|\tilde{Y}| \geq \frac{\eta}{10}$. Let $\tilde{E}=\cup_{j} \rho_{j} E$. Then $\tilde{E}$ satisfies (46) when $\nu \in \tilde{Y}$ so $|\tilde{E}| \gtrsim \lambda\left(\frac{\delta^{2}}{\eta}\right)^{\epsilon}$, hence $|E| \gtrsim \lambda M^{-1}\left(\frac{\delta^{2}}{\eta}\right)^{\epsilon}$ which is (50). 
Now we consider the Kakeya problem, and will give without detailed proof a few reductions made in [7], p. 152.

A. In order to prove (2) it suffices to prove the following inequality: let $E$ be a set in $\mathbb{R}^{n}$, let $\Omega$ be a subset of $S^{n-1}$ with $|\Omega| \geq \frac{1}{2}$, and assume that for any $e \in \Omega$ there is a tube $T_{e}^{\delta}(a)$ such that $\left|T_{e}^{\delta}(a) \cap E\right| \geq \lambda\left|T_{e}^{\delta}(a)\right|$. Then

$$
\forall \epsilon>0 \exists C_{\epsilon}:|E| \geq C_{\epsilon}^{-1} \delta^{\epsilon} \lambda^{n}
$$

To make this reduction one first observes that (2) is equivalent to the corresponding restricted weak type statement,

$$
\left|\left\{e \in S^{n-1}: f_{\delta}^{*}(e) \geq \lambda\right\}\right| \lesssim \delta^{-\epsilon} \frac{|E|}{\lambda^{n}}
$$

where $f=\chi_{E}$, and then uses the above argument from [50] to reduce (52) to the case where the left hand side is $\geq \frac{1}{2}$. Furthermore, if $\left|E \cap T_{e}^{\delta}(a)\right| \geq \lambda\left|T_{e}^{\delta}(a)\right|$ even for one choice of $e$ and $a$ then clearly $|E| \gtrsim \lambda \delta^{n-1}$. It follows that in proving (51) we can assume $\lambda \geq \delta$.

B. We define $\mathcal{Q}$ to be the unit cube $[0,1) \times \ldots \times[0,1)$. Let $N$ be an integer to be fixed below, such that $\frac{1}{N}<\delta$. If $\nu \in \mathbb{Z}^{n}$, then we define $Q_{\nu}$ to be the cube $\left[\frac{\nu_{1}}{N}, \frac{\nu_{1}+1}{N}\right) \times$ $\ldots \times\left[\frac{\nu_{n}}{N}, \frac{\nu_{n}+1}{N}\right)$. When we refer below to a $\frac{1}{N}$-cube we always mean a cube which is of the form $Q_{\nu}$ for some $\nu \in \mathbb{Z}^{n}$. In proving (51) we can assume that $E$ is contained in $\mathcal{Q}$; this follows easily since the tubes $T_{e}^{\delta}(a)$ have diameter $\lesssim 1$. Furthermore we can assume that $E$ is a union of $\frac{1}{N}$-cubes; see [7].

C. It is easy to see that $f_{\delta}^{*}\left(e^{\prime}\right) \leq C f_{\delta}^{*}(e)$ if $\left|e-e^{\prime}\right| \leq \delta$, since any tube $T_{e^{\prime}}^{\delta}(b)$ can be covered by a bounded number of tubes of the form $T_{e}^{\delta}(a)$. Accordingly if $\Omega$ is as in A., $C_{1}$ is a constant, and if $\operatorname{dist}(e, \Omega) \leq C_{1} \delta$ then there is a such that $\left|T_{e}^{\delta}(a) \cap E\right| \geq C^{-1} \lambda\left|T_{e}^{\delta}(a)\right|$ where $C$ depends on $C_{1}$.

In proving (51) we may assume that

$$
\left|\Omega \cap\left\{e \in S^{n-1}: e_{1} \geq \frac{1}{2}\right\}\right|
$$

is bounded below by a constant depending on $n$ only, since we can always achieve this by an appropriate choice of coordinates. In addition, as indicated above we may assume $\lambda \geq \delta$, and we may certainly assume that $\epsilon$ is small. Fix integers $N$ and $B$ satisfying the following relations:

$$
B^{-1} N^{2 n \epsilon} \approx \lambda \text { and } \frac{B}{N} \approx \delta
$$

Then $N \approx(\delta \lambda)^{\frac{-1}{1-2 n \epsilon}}$, so that

$$
N \delta \text { is large, } N \leq \delta^{-3}, B \text { is large, and } B N^{-n} \leq B^{-1}
$$


Define a map $\Phi: \mathbb{R}^{n} \rightarrow \mathbb{R}$ via

$$
\Phi(x)=\frac{\left[N x_{1}\right]}{N}+\frac{\left[N x_{2}\right]}{N^{2}}+\ldots+\frac{\left[N x_{n-1}\right]}{N^{n-1}}+\frac{N x_{n}}{N^{n}}
$$

Then $\Phi$ maps $\mathcal{Q}$ into $[0,1)$. We make a few additional remarks about the definition:

(i) Note the distinguished role played by the last coordinate.

(ii) $\Phi$ maps $\frac{1}{N}$-cubes on intervals of length $N^{-n}$, hence if $E$ is a union of $\frac{1}{N}$-cubes then $|\Phi(E)|=|E|$.

(iii) Suppose that $x \in \mathbb{R}^{n}$. Then $x$ belongs to a unique $\frac{1}{N}$-cube $Q_{\nu}$. Define $\tau(x)$ ("tower over $x ")$ via

$$
\tau(x)=\cup\left(Q_{\mu}: \mu_{j}=\nu_{j} \text { when } j<n \text { and }\left|\mu_{n}-\nu_{n}\right| \leq B\right)
$$

Then, for any $x, \Phi$ maps $\tau(x)$ on an interval of length $\frac{2 B+1}{N^{n}}$.

(iv) Suppose that $w=\left(\frac{k_{1}}{N}, \ldots, \frac{k_{n}}{N}\right)$ where the $\left\{k_{j}\right\}$ are integers. Set $\nu(w)=\sum_{j} \frac{k_{j}}{N^{j}}$. Then $\Phi$ maps any arithmetic progression $\{x+j w\}_{j \in \mathbb{Z}}$ to an arithmetic progression in $\mathbb{R}$ with modulus $\nu(w)$.

A lattice vector will be by definition a vector in $\mathbb{R}^{n}$ of the form

$$
w=\left(\frac{k_{1}}{N}, \ldots, \frac{k_{n}}{N}\right)
$$

where the $\left\{k_{j}\right\}$ are integers with $k_{1} \in\left(\frac{N}{2 B}, \frac{N}{B}\right)$ and $\sqrt{\sum_{j} k_{j}^{2}} \leq 2 k_{1}$. Thus any lattice vector $w$ satisfies $|w| \approx \frac{1}{B}$. We note that if $e \in S^{n-1}$ satisfies $e_{1} \geq \frac{1}{2}$ then $\left|e-\frac{w}{|w|}\right| \lesssim \delta$ for approximately $\frac{N}{B}$ lattice vectors $w$, namely all the lattice vectors $w=\frac{k}{N}$ which correspond to integer vectors $k$ such that $|k-t e| \lesssim 1$ for some $t$ with $t \approx \frac{N}{B}$. Accordingly, for an appropriate constant $A$ there are $\gtrsim\left(\frac{N}{B}\right)^{n}$ lattice vectors $w$ such that $\operatorname{dist}\left(\frac{w}{|w|}, \Omega\right) \leq A \delta$. We denote this set of lattice vectors by $\Lambda$.

If $w \in \Lambda$, then we will abuse our notation slightly and denote the tube $T_{\frac{w}{|w|}}^{\delta}(a)$ by $T_{w}^{\delta}(a)$. By C. above, for each $w \in \Lambda$ we can choose $a \in \mathbb{R}^{n}$ so that $\left|T_{w}^{\delta}(a) \cap E\right| \gtrsim \lambda\left|T_{w}^{\delta}(a)\right|$. It then follows by an averaging argument ${ }^{5}$ that there is $a^{\prime} \in \mathbb{R}^{n}$ such that

$$
\left|E \cap\left(\cup_{j=1}^{B} \tau\left(a^{\prime}+j w\right)\right)\right| \gtrsim \lambda\left|\cup_{j=1}^{B} \tau\left(a^{\prime}+j w\right)\right|
$$

\footnotetext{
${ }^{5}$ Namely: let $m$ be the measure of the set $\cup_{j=1}^{B} \tau\left(a^{\prime}+j w\right) ; m$ is clearly independent of $a^{\prime}$, and furthermore if $x \in \mathbb{R}^{n}$ is given then the measure of the set $\sigma_{x}=\left\{a^{\prime}: x \in \cup_{j=1}^{B} \tau\left(a^{\prime}+j w\right)\right\} \mid$ is also comparable to $m$. If $x \in T_{w}^{\delta}(a)$ then, since $\frac{B}{N} \lesssim \delta$ and $|w| \lesssim \frac{1}{B}$, the set $\sigma_{x}$ will be contained in $\tilde{T}_{w}^{\delta}(a)$, the dilation of $T_{w}^{\delta}(a)$ by a suitable fixed constant. It follows that $\int_{\tilde{T}_{w} \delta(a)}\left|E \cap\left(\cup_{j=1}^{B} \tau\left(a^{\prime}+j w\right)\right)\right| d a^{\prime} \geq$ $\int_{T_{w}^{\delta}(a) \cap E}\left|\sigma_{x}\right| d x \geq \lambda m\left|T_{w}^{\delta}(a)\right| \approx \lambda m\left|\tilde{T}_{w}^{\delta}(a)\right|$, so (55) holds for suitable $a^{\prime} \in \tilde{T}_{w}^{\delta}(a)$.
} 
Now set $\rho=\frac{B}{4 N^{n}}$. By (iv) above, the image of the progression $a^{\prime}, a^{\prime}+w, \cdots a^{\prime}+B w$ under $\Phi$ is an arithmetic progression $\beta, \beta+\nu(w), \cdots, \beta+B \nu(w)$. By (iii), $\Phi\left(\cup_{j=1}^{B} \tau\left(a^{\prime}+j w\right)\right)$ is a union of intervals containing the points of this progression, with the length of each interval being less than $\rho$ and comparable to $\rho$. Since $E$ and $\cup_{j=1}^{B} \tau\left(a^{\prime}+j w\right)$ are unions of $\frac{1}{N}$-cubes, (55) and (ii) then imply that $\left|\Phi(E) \cap P_{\nu}^{\rho}(\beta)\right| \gtrsim \lambda\left|P_{\nu}^{\rho}(\beta)\right|$. We conclude:

If $\nu=\nu(w)$ for some $w \in \Lambda$, then there is $\beta$ such that

$$
\left|P_{\nu}^{\rho}(\beta) \cap E\right| \gtrsim \lambda\left|P_{\nu}^{\rho}(\beta)\right|
$$

Let $Y=\left\{\nu \in \mathbb{R}:|\nu-\nu(w)| \leq N^{-n}\right.$ for some $\left.w \in \Lambda\right\}$. It follows easily that (56) continues to hold (for suitable $\beta$ ) for any $\nu \in Y$. Note that $Y \subset\left(\frac{1}{2 B}, \frac{2}{B}\right)$ (because of the requirement $\left.\frac{N}{2 B} \leq k_{1} \leq \frac{N}{B}\right)$ and also $|Y| \gtrsim B^{-n}$, since the set $\{\nu(w): w \in \Lambda\}$ is $N^{-n}$-separated and has cardinality $\gtrsim\left(\frac{N}{B}\right)^{n}$.

Now $\lambda$ is large compared with $B^{-1} \cdot\left(B\left(B N^{-n}\right)^{2}\right)^{-\epsilon}$ by (53), (54), so we can apply (50) with $\eta=B^{-1}, \delta=B N^{-n}$, and with $\frac{|Y|}{\eta} \gtrsim B^{-(n-1)}$. We conclude that $|\Phi(E)| \gtrsim$ $\lambda B^{-(n-1)}\left(B^{3} N^{-2 n}\right)^{\epsilon}$. Again using (53) and (54), we obtain $|\Phi(E)| \gtrsim \lambda^{n} N^{-2 n^{2} \epsilon} \geq \lambda^{n} \delta^{6 n^{2} \epsilon}$. But $E$ is a union of $\frac{1}{N}$-cubes so $|E|=|\Phi(E)|$, and since $\epsilon$ is arbitrary this proves (51).

\section{References}

[1] D. Alvarez, Berkeley thesis, 1997, and to appear.

[2] J. Barrionuevo, A note on the Kakeya maximal operator, Math. Research Letters 3(1996), 61-65.

[3] W. Beckner, A. Carbery, S. Semmes, F. Soria, A note on restriction of the Fourier transform to spheres, Bull. London Math. Soc. 21(1989), 394-398.

[4] A.S. Besicovitch, R. Rado, A plane set of measure zero containing circumferences of every radius, J. London Math. Soc. 43 (1968), 717-719.

[5] B. Bollobas, Graph Theory: an introductory course, Graduate Texts in Mathematics vol. 63, Springer-Verlag, 1979.

[6] J. Bourgain, Averages in the plane over convex curves and maximal operators, J. Analyse Math. 47(1986), 69-85.

[7] J. Bourgain, Besicovitch type maximal operators and applications to Fourier analysis, Geometric and Functional Analysis 1 (1991), 147-187. 
[8] J. Bourgain, $L^{p}$ estimates for oscillatory integrals in several variables, Geometric and Functional Analysis 1(1991), 321-374.

[9] J. Bourgain, On the distribution of Dirichlet sums, J. Anal. Math. 60(1993), 21-32.

[10] J. Bourgain, Some new estimates for oscillatory integrals, in Essays on Fourier analysis in honor of Elias M. Stein, ed. C. Fefferman, R. Fefferman, S. Wainger, Princeton University Press, 1994.

[11] J. Bourgain, Hausdorff dimension and distance sets, Israel J. Math 87(1994), 193201.

[12] J. Bourgain, Estimates for cone multipliers, Operator Theory: Advances and Applications, 77 (1995), 41-60.

[13] A. Carbery, Restriction implies Bochner-Riesz for paraboloids, Proc. Cambridge Phil. Soc. 111(1992), 525-529.

[14] B. Chazelle, H. Edelsbrunner, L. J. Guibas, R. Pollack, R. Seidel, M. Sharir, J. Snoeyink, Counting and cutting cycles of lines and rods in space, Comput. Geom. Theory Appls. 1(1992), 305- 323.

[15] M. Christ, Estimates for the k- plane transform, Indiana Univ. Math. J. 33(1984), 891-910.

[16] M. Christ, J. Duoandikoetxea, J. L. Rubio de Francia, Maximal operators related to the Radon transform and the Calderon- Zygmund method of rotations, Duke Math. J. 53(1986),189-209.

[17] K. L. Clarkson, H. Edelsbrunner, L. J. Guibas, M. Sharir, E. Welzl, Combinatorial complexity bounds for arrangements of curves or spheres, Discrete Comput. Geom. 5(1990), 99-160.

[18] A. Cordoba, The Kakeya maximal function and spherical summation multipliers, Amer. J. Math. 99 (1977), 1-22.

[19] R. O. Davies, Some remarks on the Kakeya problem, Proc. Cambridge Phil. Soc. 69(1971), 417-421.

[20] S. Drury, $L^{p}$ estimates for the x-ray transform, Ill. J. Math. 27(1983), 125-129.

[21] K. J. Falconer, The geometry of fractal sets, Cambridge University Press, 1985.

[22] K. J. Falconer, On the Hausdorff dimension of distance sets, Mathematika 32(1985), 206-212. 
[23] C. Fefferman, The multiplier problem for the ball, Ann. Math. 94(1971), 330-336.

[24] R. L. Graham, B. L. Rothschild, J. H. Spencer, Ramsey Theory, 2nd edition, WileyInterscience, 1990.

[25] M. de Guzman, Real variable methods in Fourier Analysis, North-Holland, 1981.

[26] N. Katz, A counterexample for maximal operators over a Cantor set of directions, Math. Research Letters 3(1996), 527-536.

[27] N. Katz, Remarks on maximal operators over arbitrary sets of directions, to appear.

[28] N. Katz, to appear.

[29] U. Keich, to appear

[30] J. R. Kinney, A thin set of circles, Amer. Math. Monthly 75 (1968), 1077-1081.

[31] L. Kolasa, T. Wolff, On some variants of the Kakeya problem, Pac. J. Math., to appear.

[32] L. Kuipers, H. Niederreiter, Uniform Distribution of Sequences, Wiley-Interscience, 1974.

[33] J. M. Marstrand, Packing planes in $\mathbb{R}^{3}$, Mathematika 26(1979), 180-183.

[34] J. M. Marstrand, Packing circles in the plane, Proc. London Math. Soc. 55 (1987), $37-58$.

[35] W. Minicozzi, C. Sogge, Negative results for Nikodym maximal functions and related oscillatory integrals in curved space, to appear.

[36] G. Mockenhoupt, A. Seeger, C. Sogge, Wave front sets and Bourgain's circular maximal theorem, Ann. Math. 134 (1992), 207-218.

[37] H. L. Montgomery, Ten lectures on the interface between analytic number theory and harmonic analysis, CBMS Regional Conference Series in Mathematics, vol. 84, American Mathematical Society, 1994.

[38] A. Moyua, A. Vargas, L. Vega, Schrodinger maximal functions and restriction properties of the Fourier transform, International Math. Res. Notices no. 16 (1996), 793-815.

[39] D. M. Oberlin, E. M. Stein, Mapping properties of the Radon transform, Indiana Univ. Math. J. 31(1982), 641-650. 
[40] J. Pach, P. Agarwal, Combinatorial Geometry, Wiley-Interscience, 1995.

[41] H. Pecher, Nonlinear small data scattering for the wave and Klein-Gordon equation, Math. Z. 185 (1984), 261-270.

[42] E. Sawyer, Families of plane curves having translates in a set of measure zero, Mathematika 34 (1987), 69-76.

[43] W. Schlag, A generalization of Bourgain's circular maximal theorem, J. Amer. Math. Soc. 10(1997), 103-122.

[44] W. Schlag, A geometric proof of the circular maximal theorem, Duke Math. J., to appear.

[45] W. Schlag, A geometric inequality with applications to the Kakeya problem in three dimensions, Geometric and Functional Analysis, to appear.

[46] W. Schlag, C. Sogge, Local smoothing estimates related to the circular maximal theorem, Math. Research Letters 4(1997), 1-15.

[47] M. Sharir, On joints in arrangements of lines in space, J. Comb. Theory A 67(1994), 89-99.

[48] C. Sogge, Propagation of singularities and maximal functions in the plane, Inv. Math. 104 (1991), 349-376.

[49] C. Sogge, Concerning Nikodym-type sets in 3-dimensional curved space, to appear.

[50] E. M. Stein, On limits of sequences of operators, Ann. Math. 74(1961), 140-170.

[51] E. M. Stein, Maximal functions: spherical means, Proc. Nat. Acad. Sci. USA 73(1976), 2174-2175.

[52] E. M. Stein, Harmonic Analysis, Princeton University Press, 1993.

[53] E. M. Stein, G. L. Weiss, Introduction to Fourier analysis on Euclidean spaces, Princeton University Press, 1971.

[54] E. M. Stein, N. J. Weiss, On the convergence of Poisson integrals, Trans. Amer. Math. Soc. 140(1969), 34-54.

[55] L. Szekely, Crossing numbers and hard Erdos problems in discrete geometry, Comb. Prob. Comput. 6(1997), 353-358.

[56] E. Szemeredi, W. T. Trotter Jr., Extremal problems in discrete geometry, Combinatorica 3(1983), 381-392. 
[57] M. Talagrand, Sur la measure de la projection d'un compact et certaines familles de cercles, Bull. Sci. Math. (2) 104 (1980), 225-231.

[58] T. Tao, The Bochner-Riesz conjecture implies the restriction conjecture, to appear.

[59] T. Tao, A. Vargas, L. Vega, A bilinear approach to the restriction and Kakeya conjectures, to appear.

[60] T. Wolff, An improved bound for Kakeya type maximal functions, Revista Math. Iberoamericana 11 (1995), 651-674.

[61] T. Wolff, A Kakeya type problem for circles, Amer. J. Math. 119(1997), 985-1026.

[62] T. Wolff, A mixed norm estimate for the x-ray transform, to appear in Revista Math. Iberoamericana. 